\title{
SOME REMARKS ON THE HYPERELLIPTIC MODULI OF GENUS 3
}

\author{
T. SHASKA
}

\begin{abstract}
In 1967, Shioda 23 determined the ring of invariants of binary octavics and their syzygies using the symbolic method. We discover that the syzygies determined in 23] are incorrect. In this paper, we compute the correct equations among the invariants of the binary octavics and give necessary and sufficient conditions for two genus 3 hyperelliptic curves to be isomorphic over an algebraically closed field $k$, char $k \neq 2,3,5,7$. For the first time, an explicit equation of the hyperelliptic moduli for genus 3 is computed in terms of absolute invariants.
\end{abstract}

\section{INTRODUCTION}

Let $k$ be an algebraically closed field. A binary form of degree $d$ is a homogeneous polynomial $f(X, Y)$ of degree $d$ in two variables over $k$. Let $V_{d}$ be the $k$-vector space of binary forms of degree $d$. The group $G L_{2}(k)$ of invertible $2 \times 2$ matrices over $k$ acts on $V_{d}$ by coordinate change. Many problems in algebra involve properties of binary forms which are invariant under these coordinate changes. In particular, any hyperelliptic genus $g$ curve over $k$ has a projective equation of the form $Z^{2} Y^{2 g}=f(X, Y)$, where $f$ is a binary form of degree $d=2 g+2$ and non-zero discriminant. Two such curves are isomorphic if and only if the corresponding binary forms are conjugate under $G L_{2}(k)$. Therefore the moduli space $\mathcal{H}_{g}$ of hyperelliptic genus $g$ curves is the affine variety whose coordinate ring is the ring of $G L_{2}(k)$-invariants in the coordinate ring of the set of elements of $V_{d}$ with non-zero discriminant. It is well known that the moduli spaces $\mathcal{H}_{g}$ of hyperelliptic curves of genus $g, g \neq 4$, are all rational varieties, i.e. isomorphic to a purely transcendental extension field $k\left(t_{1}, \ldots, t_{r}\right)$; see Igusa [9, Katsylo [10].

Generators for this and similar invariant rings in lower degree were constructed by Clebsch, Bolza and others in the last century using complicated symbolic calculations. For the case of sextics, Igusa 9] extended this to algebraically closed fields of any characteristic using difficult techniques of algebraic geometry. For a modern treatment of the degree six case see [11].

The case of binary octavics has been first studied during the 19th century by von Gall [24 and Alagna 1,2, Shioda in his thesis [23] determined the structure of the ring of invariants $\mathcal{R}_{8}$, which turns out to be generated by nine $S L(2, k)$-invariants $J_{2}, \cdots, J_{10}$ satisfying five algebraic relations. He computed explicitly these five syzygies, and determined the corresponding syzygy-sequence and therefore the structure of the ring $\mathcal{R}_{8}$; see Shioda [23].

This paper started as a project to implement an algorithm which determines if two genus 3 hyperelliptic curves are isomorphic over $\mathbb{C}$. According to Shioda [23, Thm. 5]; two genus 3 hyperelliptic curves are isomorphic if and only if the corresponding 9-tuples $\left(J_{2}, \ldots, J_{10}\right)$ are equivalent, satisfying five syzygies $R_{i}\left(J_{2}, \ldots, J_{10}\right)=0$, for $i=1, \ldots, 5$ and non-zero discriminant $\Delta \neq 0$. While trying to implement the syzygies $R_{i}\left(J_{2}, \ldots, J_{10}\right)=0$, for $i=1, \ldots, 5$ we discovered that they are not satisfied for a generic octavic. Hence, such algebraic relations in terms of $J_{2}, \ldots, J_{10}$ are incorrect as stated in 23]; cf Example 1.

Indeed, if you take any random binary octavics then its invariants will not satisfy the Shioda's relations. Since the results in [23] do not hold, then one needs to determine explicitly the algebraic relations between the invariants in order to have an explicit description of the ring of invariants $\mathcal{R}_{8}$ and its field of fractions $\mathcal{S}_{8}$. This will be our goal for the rest of this paper.

In section 2, we give some basic preliminaries on invariants of binary forms. In section 3 , we define the main invariants of binary octavics via transvectants. The definitions are the same as used by classical invariant theorists, however, we scale be a constant factor in order to work with primitive polynomials with integer coefficients. We show an example of a binary form which does not satisfy the syzygies as claimed in [23]; see Example 1] Furthermore,

2000 Mathematics Subject Classification. Primary 54C40, 14E20; Secondary 46E25, 20C20.

Key words and phrases. invariants, binary forms, genus 3, algebraic curves.

The author was supported in part by NSA Grant \#000000. 
we determine the algebraic relations between the invariants $J_{2}, \ldots, J_{10}$. Such algebraic relations determine the ring of invariants $\mathcal{R}_{8}$.

From the basic $S L(2, k)$-invariants $J_{2}, \ldots, J_{8}$ we define six $G L(2, k)$-invariants

$$
t_{1}:=\frac{J_{3}^{2}}{J_{2}^{3}}, \quad t_{2}:=\frac{J_{4}}{J_{2}^{2}}, \quad t_{3}:=\frac{J_{5}}{J_{2} \cdot J_{3}}, \quad t_{4}:=\frac{J_{6}}{J_{2} \cdot J_{4}}, \quad t_{5}:=\frac{J_{7}}{J_{2} \cdot J_{5}}, \quad t_{6}:=\frac{J_{8}}{J_{2}^{4}},
$$

which we call absolute invariants. There is an algebraic relation

$$
T\left(i_{1}, \ldots, t_{6}\right)=0
$$

that such invariants satisfy, computed for the first time. Shioda in his paper talked about this relation but never attempted to compute it. It has total degree 14 , degrees $5,10,6,6,5,5$ in $t_{1}, \ldots, t_{6}$ respectively, and has 25 464 monomials. The field of invariants $\mathcal{S}_{8}$ of binary octavics is $\mathcal{S}_{8}=k\left(t_{1}, \ldots, t_{6}\right)$, where $t_{1}, \ldots, t_{6}$ satisfy the equation $T\left(t_{1}, \ldots, t_{6}\right)=0$. Hence, we have an explicit description of the hyperelliptic moduli $\mathcal{H}_{3}$. A birational parametrization of this variety seems out of reach computationally.

All of our results are implemented in a Maple package and made available at [19]. Such results will be helpful in the arithmetic of genus 3 hyperelliptic curves. The computation of the equation in (19) makes now possible to describe the subloci of $\mathcal{H}_{3}$ in terms of the $t_{1}, \ldots, t_{6}$ invariants and other problems on genus 3 hyperelliptic curves as described in [5, 6, $13,18,20,22$ among others.

\section{Preliminaries on InVARIANTS OF BINARY FORMS}

In this section we define the action of $G L_{2}(k)$ on the space of binary forms and discuss the basic notions of their invariants. Most of this section is a summary of section 2 in [1]. Throughout this section $k$ denotes an algebraically closed field.

2.1. Action of $G L_{2}(k)$ on binary forms. Let $k[X, Y]$ be the polynomial ring in two variables and let $V_{d}$ denote the $(d+1)$-dimensional subspace of $k[X, Y]$ consisting of homogeneous polynomials.

$$
f(X, Y)=a_{0} X^{d}+a_{1} X^{d-1} Y+\ldots+a_{d} Y^{d}
$$

of degree $d$. Elements in $V_{d}$ are called binary forms of degree $d$. We let $G L_{2}(k)$ act as a group of automorphisms on $k[X, Y]$ as follows: if

$$
g=\left(\begin{array}{ll}
a & b \\
c & d
\end{array}\right) \in G L_{2}(k)
$$

then

$$
g\left(\begin{array}{l}
X \\
Y
\end{array}\right)=\left(\begin{array}{l}
a X+b Y \\
c X+d Y
\end{array}\right)
$$

This action of $G L_{2}(k)$ leaves $V_{d}$ invariant and acts irreducibly on $V_{d}$.

Remark 1. It is well known that $S L_{2}(k)$ leaves a bilinear form (unique up to scalar multiples) on $V_{d}$ invariant. This form is symmetric if $d$ is even and skew symmetric if $d$ is odd.

Let $A_{0}, A_{1}, \ldots, A_{d}$ be coordinate functions on $V_{d}$. Then the coordinate ring of $V_{d}$ can be identified with $k\left[A_{0}, \ldots, A_{d}\right]$. For $I \in k\left[A_{0}, \ldots, A_{d}\right]$ and $g \in G L_{2}(k)$, define $I^{g} \in k\left[A_{0}, \ldots, A_{d}\right]$ as follows

$$
I^{g}(f)=I(g(f))
$$

for all $f \in V_{d}$. Then $I^{g h}=\left(I^{g}\right)^{h}$ and (3) defines an action of $G L_{2}(k)$ on $k\left[A_{0}, \ldots, A_{d}\right]$.

Definition 1. Let $\mathcal{R}_{d}$ be the ring of $S L_{2}(k)$ invariants in $k\left[A_{0}, \ldots, A_{d}\right]$, i.e., the ring of all $I \in k\left[A_{0}, \ldots, A_{d}\right]$ with $I^{g}=I$ for all $g \in S L_{2}(k)$.

Note that if $I$ is an invariant, so are all its homogeneous components. So $\mathcal{R}_{d}$ is graded by the usual degree function on $k\left[A_{0}, \ldots, A_{d}\right]$.

Since $k$ is algebraically closed, the binary form $f(X, Y)$ in Eq. (1) can be factored as

$$
f(X, Y)=\left(y_{1} X-x_{1} Y\right) \ldots\left(y_{d} X-x_{d} Y\right)=\prod_{1 \leq i \leq d} \operatorname{det}\left(\begin{array}{ll}
X & x_{i} \\
Y & y_{i}
\end{array}\right)
$$


The points with homogeneous coordinates $\left(x_{i}, y_{i}\right) \in \mathbb{P}^{1}$ are called the roots of the binary form in Eq. (1). Thus for $g \in G L_{2}(k)$ we have

where

$$
g(f(X, Y))=(\operatorname{det}(g))^{d}\left(y_{1}^{\prime} X-x_{1}^{\prime} Y\right) \ldots\left(y_{d}^{\prime} X-x_{d}^{\prime} Y\right) .
$$

$$
\left(\begin{array}{l}
x_{i}^{\prime} \\
y_{i}^{\prime}
\end{array}\right)=g^{-1}\left(\begin{array}{l}
x_{i} \\
y_{i}
\end{array}\right)
$$

Definition 2. The nullcone $\mathcal{N}_{d}$ of $V_{d}$ is the zero set of all homogeneous elements in $\mathcal{R}_{d}$ of positive degree

The notion of nullcone was first used by Hilbert; see [8]. Next we define the Reynold's operator on $k\left[A_{0}, \ldots, A_{d}\right]$.

Lemma 1. Let char $(k)=0$ and $\Omega_{s}$ be the subspace of $k\left[A_{0}, \ldots, A_{d}\right]$ consisting of homogeneous elements of degree s. Then there is a k-linear map

$$
R: k\left[A_{0}, \ldots, A_{d}\right] \rightarrow \mathcal{R}_{d}
$$

with the following properties:

(a) $R\left(\Omega_{s}\right) \subseteq \Omega_{s}$ for all $s$

(b) $R(I)=I$ for all $I \in \mathcal{R}_{d}$

(c) $R(g(f))=R(f)$ for all $f \in k\left[A_{0}, \ldots, A_{d}\right]$

Proof. $\Omega_{s}$ is a polynomial module of degree $s$ for $S L_{2}(k)$. Since $S L_{2}(k)$ is linearly reductive in $\operatorname{char}(k)=0$, there exists a $S L_{2}(k)$-invariant subspace $\Lambda_{s}$ of $\Omega_{s}$ such that $\Omega_{s}=\left(\Omega_{s} \cap \mathcal{R}_{d}\right) \bigoplus \Lambda_{s}$. Define

$$
R: k\left[A_{0}, \ldots, A_{d}\right] \rightarrow \mathcal{R}_{d}
$$

such that $R\left(\Lambda_{s}\right)=0$ and $R_{\mid \Omega_{s} \cap \mathcal{R}_{d}}=i d$. Then $R$ is $k$-linear and the rest of the proof is clear from the definition of $R$.

The map $R$ is called the Reynold's operator.

Lemma 2. Suppose char $(k)=0$. Then every maximal ideal in $\mathcal{R}_{d}$ is contained in a maximal ideal of $k\left[A_{0}, \ldots, A_{d}\right]$.

Proof. If $\mathcal{I}$ is a maximal ideal in $\mathcal{R}_{d}$ which generates the unit ideal of $k\left[A_{0}, \ldots, A_{d}\right]$, then there exist $m_{1}, m_{2}, \ldots$ ,$m_{t} \in \mathcal{I}$ and $f_{1}, f_{2}, \ldots, f_{t} \in k\left[A_{0}, \ldots, A_{d}\right]$ such that

$$
1=m_{1} f_{1}+\ldots+m_{t} f_{t}
$$

Applying the Reynold's operator to the above equation we get

$$
1=m_{1} R\left(f_{1}\right)+\ldots+m_{t} R\left(f_{t}\right)
$$

But $R\left(f_{i}\right) \in \mathcal{R}_{d}$ for all $i$. This implies $1 \in \mathcal{I}$, a contradiction.

Theorem 1. [Hilbert's Finiteness Theorem] Suppose char $(k)=0$. Then $\mathcal{R}_{d}$ is finitely generated over $k$.

Proof. Let $\mathcal{I}_{0}$ be the ideal in $k\left[A_{0}, \ldots, A_{d}\right]$ generated by all homogeneous invariants of positive degree. Because $k\left[A_{0}, \ldots, A_{d}\right]$ is Noetherian, there exist finitely many homogeneous elements $J_{1}, \ldots \quad J_{r}$ in $\mathcal{R}_{d}$ such that $\mathcal{I}_{0}=$ $\left(J_{1}, \ldots, J_{r}\right)$. We prove $\mathcal{R}_{d}=k\left[J_{1}, \ldots, J_{r}\right]$. Let $J \in \mathcal{R}_{d}$ be homogeneous of degree $d$. We prove $J \in k\left[J_{1}, \ldots, J_{r}\right]$ using induction on $d$. If $d=0$, then $J \in k \subset k\left[J_{1}, \ldots, J_{r}\right]$. If $d>0$, then

$$
J=f_{1} J_{1}+\ldots+f_{r} J_{r}
$$

with $f_{i} \in k\left[A_{0}, \ldots, A_{d}\right]$ homogeneous and $\operatorname{deg}\left(f_{i}\right)<d$ for all $i$. Applying the Reynold's operator to Eq. (6) we have

$$
J=R\left(f_{1}\right) J_{1}+\ldots+R\left(f_{r}\right) J_{r}
$$

then by lemma 1, we have $R\left(f_{i}\right)$ is a homogeneous element in $\mathcal{R}_{d}$ with $\operatorname{deg}\left(R\left(f_{i}\right)\right)<d$ for all $i$ and hence by induction we have $R\left(f_{i}\right) \in k\left[J_{1}, \ldots, J_{r}\right]$ for all $i$. Thus $J \in k\left[J_{1}, \ldots, J_{r}\right]$.

If $k$ is of arbitrary characteristic, then $S L_{2}(k)$ is geometrically reductive, which is a weakening of linear reductivity; see Haboush [7. It suffices to prove Hilbert's finiteness theorem in any characteristic; see Nagata [12. The following theorem is also due to Hilbert [8].

Theorem 2. Let $I_{1}, I_{2}, \ldots, I_{s}$ be homogeneous elements in $\mathcal{R}_{d}$ whose common zero set equals the null cone $\mathcal{N}_{d}$. Then $\mathcal{R}_{d}$ is finitely generated as a module over $k\left[I_{1}, \ldots, I_{s}\right]$. 
Proof. (i) $\operatorname{char}(k)=0$ : By theorem 1, we have $\mathcal{R}_{d}=k\left[J_{1}, J_{2}, \ldots, J_{r}\right]$ for some homogeneous invariants $J_{1}, \ldots, J_{r}$. Let $\mathcal{I}_{0}$ be the maximal ideal in $\mathcal{R}_{d}$ generated by all homogeneous elements in $\mathcal{R}_{d}$ of positive degree. Then the theorem follows if $I_{1}, \ldots, I_{s}$ generate an ideal $\mathcal{I}$ in $\mathcal{R}_{d}$ with $\operatorname{rad}(\mathcal{I})=\mathcal{I}_{0}$. For if this is the case, we have an integer $q$ such that

$$
J_{i}^{q} \in \mathcal{I} \forall i
$$

Set $S:=\left\{J_{1}^{i_{1}} J_{2}^{i_{2}} \ldots J_{r}^{i_{r}}: 0 \leq i_{1}, \ldots, i_{r}<q\right\}$. Let $\mathcal{M}$ be the $k\left[I_{1}, \ldots, I_{s}\right]$-submodule in $\mathcal{R}_{d}$ generated by $S$. We prove $\mathcal{R}_{d}=\mathcal{M}$. Let $J \in \mathcal{R}_{d}$ be homogeneous. Then $J=J^{\prime}+J^{\prime \prime}$ where $J^{\prime} \in \mathcal{M}, J^{\prime \prime}$ is a $k$-linear combination of $J_{1}^{i_{1}} J_{2}^{i_{2}} \ldots J_{r}^{i_{r}}$ with at least one $i_{\nu} \geq q$ and $\operatorname{deg}(J)=\operatorname{deg}\left(J^{\prime}\right)=\operatorname{deg}\left(J^{\prime \prime}\right)$. Hence (7) implies $J^{\prime \prime} \in \mathcal{I}$ and so we have

$$
J^{\prime \prime}=f_{1} I_{1}+\cdots+f_{s} I_{s},
$$

where $f_{i} \in \mathcal{R}_{d}$ for all $i$. Then $\operatorname{deg}\left(f_{i}\right)<\operatorname{deg}\left(J^{\prime \prime}\right)=\operatorname{deg}(J)$ for all $i$. Now by induction on degree of $J$ we may assume $f_{i} \in \mathcal{M}$ for all $i$. This implies $J^{\prime \prime} \in \mathcal{M}$ and hence $J \in \mathcal{M}$. Therefore $\mathcal{M}=\mathcal{R}_{d}$. So it only remains to prove $\operatorname{rad}(\mathcal{I})=\mathcal{I}_{0}$. This follows from Hilbert's Nullstellensatz and the following claim.

Claim: $\mathcal{I}_{0}$ is the only maximal ideal containing $I_{1}, \ldots, I_{s}$.

Suppose $\mathcal{I}_{1}$ is a maximal ideal in $\mathcal{R}_{d}$ with $I_{1}, \ldots, I_{s} \in \mathcal{I}_{1}$. Then from Lemma 2 we know there exists a maximal ideal $\mathcal{J}$ of $k\left[A_{0}, \ldots, A_{d}\right]$ with $\mathcal{I}_{1} \subset \mathcal{J}$. The point in $V_{d}$ corresponding to $\mathcal{J}$ lies on the null cone $\mathcal{N}_{d}$ because $I_{1}, \ldots$ ,I $I_{s}$ vanish on this point. Therefore $\mathcal{I}_{0} \subset \mathcal{J}$, by definition of $\mathcal{N}_{d}$. Therefore $\mathcal{J} \cap \mathcal{R}_{d}$ contains both the maximal ideals $\mathcal{I}_{1}$ and $\mathcal{I}_{0}$. Hence $\mathcal{I}_{1}=\mathcal{J} \cap \mathcal{R}_{d}=\mathcal{I}_{0}$.

(ii) $\operatorname{char}(k)=p$ : The same proof works if lemma 2 holds. Geometrically this means the morphism $\pi: V_{d} \rightarrow V_{d}$ // $S L_{2}(k)$ corresponding to the inclusion $\mathcal{R}_{d} \subset k\left[A_{0}, \ldots, A_{d}\right]$ is surjective. Here $V_{d} / / S L_{2}(k)$ denotes the affine variety corresponding to the ring $\mathcal{R}_{d}$ and is called the categorical quotient. $\pi$ is surjective because $S L_{2}(k)$ is geometrically reductive. The proof is by reduction modulo $p$; see Geyer [3].

2.2. Hyperelliptic curves of genus 3. In this section we want to use the projective equivalence of binary octavics in order two give conditions that two hyperelliptic curves of genus 3 are isomorphic.

Denote a binary form of order $2 g+2$ by

$$
f(X, Y)=\sum_{i=0}^{2 g+2} a_{i} X^{i} Y^{2 g+2-i}
$$

To each $f(X, Y)$ with no multiple roots we associate the non-singular hyperelliptic curve $C_{f}$ with affine equation $Z^{2}=f(X, 1)$. Every hyperelliptic curve of genus $g$ is obtained this way.

Two hyperelliptic curves $C_{f}$ and $C_{h}$ are birationally equivalent if and only if $f(X, Y)$ and $h(X, Y)$ are projectively equivalent, i.e., there exists a $\tau \in S L_{2}(k)$ and $\lambda \in k \backslash\{0\}$ such that $f^{\tau}=\lambda \cdot h$.

Let $\Delta_{f}$ denote the discriminant of the polynomial $f(X, 1)$. It is an invariant of degree $2(2 g+1)$. When $g=3$ then the discriminant has degree 14 and is given as a polynomial in $J_{2}, \ldots, J_{8}$.

\section{Projective inVariance of Binary octavics.}

Throughout this section char $(k) \neq 2,3,5,7$.

3.1. Covariants and invariants of binary octavics. We will use the symbolic method of classical theory to construct covariants of binary octavics. They were first constructed by van Gall who showed that there are 70 such covariants; see von Gall 24. First we recall some facts about the symbolic notation. Let

$$
f(X, Y):=\sum_{i=0}^{n}\left(\begin{array}{c}
n \\
i
\end{array}\right) a_{i} X^{n-i} Y^{i}, \quad \text { and } \quad g(X, Y):=\sum_{i=0}^{m}\left(\begin{array}{c}
m \\
i
\end{array}\right) b_{i} X^{n-i} Y^{i}
$$

be binary forms of degree $n$ and $m$ respectively. We define the $r$-transvection

$$
(f, g)^{r}:=\frac{(m-r) !(n-r) !}{n ! m !} \sum_{k=0}^{r}(-1)^{k}\left(\begin{array}{l}
r \\
k
\end{array}\right) \cdot \frac{\partial^{r} f}{\partial X^{r-k} \partial Y^{k}} \cdot \frac{\partial^{r} g}{\partial X^{k} \partial Y^{r-k}},
$$

see Grace and Young [4 for details. 
The following result gives relations among the invariants of binary forms and it is known as the Gordon's formula. It is the basis for most of the classical papers on invariant theory.

Theorem 3 (Gordon). Let $\phi_{i}, i=0,1,2$ be covariants of order $m_{i}$ and $e_{i}$ be three non-negative integers such that $e_{i}+e_{j} \leq m_{k}$ for distinct $i, j, k$. The following is true:

$$
\sum_{i} \frac{C_{i}^{e_{1}} \cdot C_{i}^{m_{1}-e_{0}-e_{2}}}{C_{i}^{m_{0}+m_{1}+1-2 e_{2}-i}}\left(\left(\phi_{0} \phi_{1}\right)^{e_{2}+1}, \phi_{2}\right)^{e_{0}+e_{1}-i}=\sum_{i} \frac{C_{i}^{e_{2}} \cdot C_{i}^{m_{2}-e_{0}-e_{1}}}{C_{i}^{m_{0}+m_{2}+1-2 e_{1}-i}}\left(\left(\phi_{0} \phi_{2}\right)^{e_{1}+1}, \phi_{1}\right)^{e_{0}+e_{2}-i}
$$

where $e_{0}=0$ or $e_{1}+e_{2}=m_{0}$.

This result has been used by many XIX century mathematicians to compute algebraic relations among invariants, most notably by Bolza for binary sextics and by Alagna for binary octavics. It provides algebraic relations among the invariants in a very similar manner that the Frobenious identities do for theta functions of hyperelliptic curves. Whether there exists some explicit relation among both formulas seems to be unknown.

For the rest of this paper $f(X, Y)$ denotes a binary octavic as below:

$$
f(X, Y)=\sum_{i=0}^{8} a_{i} X^{i} Y^{8-i}=\sum_{i=0}^{8}\left(\begin{array}{c}
n \\
i
\end{array}\right) b_{i} X^{i} Y^{n-i}
$$

where $b_{i}=\frac{(n-i) ! i !}{n !} \cdot a_{i}$, for $i=0, \ldots, 8$. We define the following covariants:

$$
g=(f, f)^{4}, \quad k=(f, f)^{6}, \quad h=(k, k)^{2}, \quad m=(f, k)^{4}, \quad n=(f, h)^{4}, \quad p=(g, k)^{4}, \quad q=(g, h)^{4} .
$$

Then, the following

$$
\begin{array}{ll}
J_{2}=2^{2} \cdot 5 \cdot 7 \cdot(f, f)^{8}, & J_{3}=\frac{1}{3} \cdot 2^{4} \cdot 5^{2} \cdot 7^{3} \cdot(f, g)^{8}, \\
J_{4}=2^{9} \cdot 3 \cdot 7^{4} \cdot(k, k)^{4}, & J_{5}=2^{9} \cdot 5 \cdot 7^{5} \cdot(m, k)^{4}, \\
J_{6}=2^{14} \cdot 3^{2} \cdot 7^{6} \cdot(k, h)^{4}, & J_{7}=2^{14} \cdot 3 \cdot 5 \cdot 7^{7} \cdot(m, h)^{4}, \\
J_{8}=2^{17} \cdot 3 \cdot 5^{2} \cdot 7^{9} \cdot(p, h)^{4}, & J_{9}=2^{19} \cdot 3^{2} \cdot 5 \cdot 7^{9} \cdot(n, h)^{4}, \\
J_{10}=2^{22} \cdot 3^{2} \cdot 5^{2} \cdot 7^{11}(q, h)^{4} &
\end{array}
$$

are $S L_{2}(k)$ - invariants. Notice that we are scaling such invariants up to multiplication by a constant for computational purposes only. We display only the first two of such invariants to avoid any confusion in the definitions

$$
\begin{aligned}
J_{2}= & 280 a_{8} a_{0}-35 a_{7} a_{1}+10 a_{6} a_{2}-5 a_{5} a_{3}+2 a_{4}{ }^{2} \\
J_{3}= & 1050 a_{8} a_{2}{ }^{2}+1050 a_{6}{ }^{2} a_{0}+75 a_{6} a_{3}{ }^{2}+75 a_{5}{ }^{2} a_{2}+12 a_{4}{ }^{3}+3920 a_{8} a_{4} a_{0}-2450 a_{8} a_{3} a_{1}+735 a_{7} a_{4} a_{1} \\
& -2450 a_{7} a_{5} a_{0}-175 a_{7} a_{3} a_{2}-110 a_{6} a_{4} a_{2}-175 a_{6} a_{5} a_{1}-45 a_{5} a_{4} a_{3}
\end{aligned}
$$

In other words, we take the numerator of the corresponding transvectants since we prefer to work over $\mathbb{Z}$ instead of $\mathbb{Q}$ and then take the primitive part of each invariant. Hence, we have $J_{i} \in \mathbb{Z}\left[a_{0}, \ldots, a_{8}\right]$, for $i=2, \ldots, 8$ and $J_{i}$ 's are primitive polynomials. In [23] such scaling is not done and this invariants are homogenous polynomials with coefficients in $\mathbb{Q}\left[a_{0}, \ldots, a_{8}\right]$ and not primitive.

Lemma 3. For each binary octavic $f(X, Y)$, its invariants defined in Eq.(10) are primitive homogeneous polynomials $J_{i} \in \mathbb{Z}\left[a_{0}, \ldots, a_{8}\right]$ of degree $i$, for $i=2, \ldots, 10$. Let $f^{\prime}=g(f)$, where

$$
g=\left(\begin{array}{ll}
a & b \\
c & d
\end{array}\right) \in G L_{2}(k)
$$

and denote the corresponding $J_{2}, \ldots, J_{10}$ of $f^{\prime}$ by $J_{2}^{\prime}, \ldots, J_{10}^{\prime}$. Then,

$$
J_{i}^{\prime}=\left(\Delta^{4}\right)^{i} J_{i}
$$

where $\Delta=a d-b c$ and $i=2, \ldots, 10$.

Proof. The first claim is immediate from the definition of the covariants and invariants. Let $f$ and $f^{\prime}$ be two binary octavics as in the hypothesis. One can check the result computationally. 
Remark 2. There are 68 invariants defined this way as discovered by van Gall 24,25] in 1880. Indeed, van Gall claimed 70 such invariants, but as discovered in XX-century there are only 68 of them. Perhaps, one that needs to be mentioned is $J_{14}$ which is the discriminant of the binary octavic.

In a couple of papers in 1892 and 1896 R. Alagna determined the algebraic relations among such invariants; see [1,2] for details. All these works have computational mistakes and are almost impossible to check.

Next we want to show that the ring of invariants $\mathcal{R}_{8}$ is finitely generated as a module over $k\left[J_{2}, \ldots, J_{7}\right]$. First we need some auxiliary lemmas.

Lemma 4. If $J_{i}=0$, for $i=2, \ldots 7$, then the $f(X, Y)$ has a multiple root.

Proof. Compute $J_{i}=0$, for $i=2, \ldots 7$. These equations imply that

$$
\operatorname{Res}\left(f(X, 1), f^{\prime}(X, 1), X\right)=0
$$

where $f^{\prime}$ is the derivative of $f$. This proves the lemma.

Theorem 4. The following hold true for any octavic.

i) An octavic has a root of multiplicity exactly four if and only if the basic invariants take the form

$$
\begin{gathered}
J_{2}=2 \cdot r^{2}, \quad J_{3}=2^{2} \cdot 3 \cdot r^{3}, \quad J_{4}=2^{6} \cdot r^{4}, \quad J_{5}=2^{6} \cdot r^{5}, \\
J_{6}=2^{9} \cdot r^{6}, \quad J_{7}=2^{9} \cdot r^{7}, \quad J_{8}=2^{11} \cdot 3^{2} \cdot r^{8},
\end{gathered}
$$

for some $r \neq 0$. Moreover, if the octavic has equation

$$
f(x, y)=x^{4}\left(a x^{4}+b x^{3} y+c x^{2} y^{2}+d x y^{3}+e y^{4}\right),
$$

then $r=e$.

ii) An octavic has a root of multiplicity 5 if and only if

$$
J_{i}=0, \text { for } i=2, \ldots, 8 .
$$

Proof. i) Let

$$
f(X, Y)=a_{0} X^{8}+a_{7} X^{7} Y+\cdots+a_{8} Y^{8}
$$

be an octavic with a root of multiplicity four. Let this root be at $(1,0)$. Then,

$$
f(X, Y)=\left(a_{4} X^{4}+a_{3} X^{3} Y+a_{2} X^{2} Y^{2}+a_{1} X Y^{3}+a_{0} Y^{4}\right) X^{4}
$$

Thus, for $r=a_{4}, J_{i}$ for $i=2, \ldots, 8$ are as claimed.

Conversely assume that Eq. (17) holds. Then, we have a multiple root. We assume the multiple root is at $(1,0)$. If this is the only root then $r=0$. Thus, there is at least one more root. We assume the other root is $(0,1)$. Then the octavic takes the form

$$
f(X, Y)=a_{2} X^{6} Y^{2}+a_{3} X^{5} Y^{3}+a_{4} X^{4} Y^{4}+a_{5} X^{3} Y^{5}+a_{6} X^{2} Y^{6}+a_{7} X Y^{7}
$$

and (17) becomes a system of six equations. We eliminate $a_{2}, a_{3}$ to get that $a_{5}=0$ or $a_{4}=r$. If $a_{4}=r$ and $a_{5} \neq 0$ then $a_{2}=a_{3}=0$ and $(1,0)$ is a root of multiplicity four. If $a_{5}=0$ then from the system we get $a_{2}=0$ or $a_{6}=0$. In both cases we have a root of multiplicity four.

ii) Suppose $(1,0)$ is a root of multiplicity 5 . Then, as in previous lemma we can take $a_{8}=a_{7}=a_{6}=a_{5}=a_{4}=0$. Then by a lemma of Hilbert [ $\left[\right.$ ] or by simple computation we have $J_{i}=0$, for $i=2, \ldots, 7$.

For the converse, since $J_{14}=0$, there is a multiple root. If there is no root other than the multiple root, we are done. Otherwise, let the multiple root be at $(1,0)$ and the other root be at $(0,1)$. Then as in the previous lemma, the octavic becomes

$$
f(X, Y)=a_{2} X^{6} Y^{2}+a_{3} X^{5} Y^{3}+a_{4} X^{4} Y^{4}+a_{5} X^{3} Y^{5}+a_{6} X^{2} Y^{6}+a_{7} X Y^{7}
$$

Compute all $J_{2}, \ldots J_{7}$. From the corresponding system of equations we can eliminate $a_{2}, a_{3}, a_{7}$. We have a few cases:

$$
a_{4}\left(-2 a_{4} a_{6}+a_{5}^{2}\right)\left(-34 a_{4} a_{6}+15 a_{5}{ }^{2}\right)\left(5476 a_{6}{ }^{2} a_{4}{ }^{2}+2025 a_{5}{ }^{4}-6780 a_{4} a_{5}{ }^{2} a_{6}\right)=0
$$

Careful analysis of each case leads to the existence of a root of multiplicity 5 . The proof is computational and we skip the details.

Remark 3. An alternative proof of the above can provided using the $k$-th subresultants of $f$ and its derivatives. Two forms have $k$ roots in common if and only if the first $k$ subresultants vanish. This is equivalent to $J_{2}=\cdots=J_{7}=0$. 


\subsection{The Null Cone of $V_{8}$ and Algebraic Dependencies.}

Theorem 5. $\mathcal{R}_{8}$ is finitely generated as a module over $k\left[J_{2}, \ldots, J_{7}\right]$.

Proof. By Theorem 2 we only have to prove $\mathcal{N}_{8}=V\left(J_{2}, \ldots, J_{7}\right)$. For $\lambda \in k^{*}$, set

$$
g(\lambda):=\left(\begin{array}{cc}
\lambda^{-1} & 0 \\
0 & \lambda
\end{array}\right)
$$

Suppose $J_{2}, \ldots, J_{7}$ vanish on an octavic $f \in V_{8}$. Then we know from Theorem 4 that $f$ has a root of multiplicity at least 5 . Let this multiple root be $(1,0)$. Then $f$ is of the form

$$
f(X, Y)=\left(a_{5} X^{3}+a_{6} X^{2} Y+a_{7} X Y^{2}+a_{8} Y^{3}\right) Y^{5}
$$

If $I \in \mathcal{R}_{8}$ is homogeneous of degree $s>0$, then

$$
I\left(f^{g(\lambda)}\right)=\lambda^{2 s} I\left(a_{5} X^{3} Y^{5}+\lambda^{2} a_{6} X^{2} Y^{6}+\lambda^{3} a_{7} X Y^{7}+\lambda^{4} a_{8} Y^{8}\right)
$$

Thus $I\left(f^{g(\lambda)}\right)$ is a polynomial in $\lambda$ with no constant term. But since $I$ is an $S L_{2}(k)$-invariant, we have $I\left(f^{g(\lambda)}\right)=$ $I(f)$ for all $\lambda$. Thus $I(f)=0$. Then, $\mathcal{N}_{8}=V\left(J_{2}, J_{3}, J_{4}, J_{5}, J_{6}, J_{7}\right)$. This completes the proof.

The above lemma is proven by Shioda in a more computational way using the symbolic method; see below for more details.

Corollary 1. $J_{2}, \ldots, J_{7}$ are algebraically independent over $k$ because $\mathcal{R}_{8}$ is the coordinate ring of the 5-dimensional variety $V_{8} / / S L_{2}(k)$.

3.2.1. Shioda's computations. The algebraic relations between $J_{2}, \ldots, J_{10}$ were computed by Shioda in 23 using the symbolic method. However, we could not confirm the correctness of such results with our computations. For a binary octavic

$$
f(X, Y)=\sum_{i=0}^{8} a_{i} X^{i} Y^{8-i}
$$

Shioda invariants are defined as

$$
\begin{aligned}
\tilde{J}_{2}= & 2 a_{8} a_{0}-16 a_{7} a_{1}+56 a_{6} a_{2}-112 a_{5} a_{3}+70 a_{4}{ }^{2} \\
\tilde{J}_{3}= & \frac{9}{392} a_{8} a_{2}{ }^{2}+\frac{9}{392} a_{6}{ }^{2} a_{0}+\frac{9}{5488} a_{6} a_{3}^{2}+\frac{9}{5488} a_{5}^{2} a_{2}+\frac{9}{560} a_{7} a_{4} a_{1}-\frac{3}{56} a_{7} a_{5} a_{0} \\
& -\frac{3}{784} a_{7} a_{3} a_{2}-\frac{33}{13720} a_{6} a_{4} a_{2}-\frac{3}{784} a_{6} a_{5} a_{1}-\frac{27}{27440} a_{5} a_{4} a_{3}+\frac{3}{35} a_{8} a_{4} a_{0}-\frac{3}{56} a_{8} a_{3} a_{1}+\frac{9}{34300} a_{4}{ }^{3}
\end{aligned}
$$

Notice that that definition of $J_{2}$ looks different from that of Shioda [23, page 1037], but that is because there $J_{2}$ is evaluated for $f(X, Y)=\sum_{i=0}^{8}\left(\begin{array}{c}8 \\ i\end{array}\right) a_{i} X^{i} Y^{8-i}$.

Now we are ready to show that the syzygies in [23, Th. 5] are not correct. Below is an example of a genus 3 hyperelliptic curve with invariants which do not satisfy Shioda relations.

Example 1. Let a genus 3 hyperelliptic curve be given by the equation

$$
y^{2}=x^{8}+x^{7}+x^{6}+x^{5}+x^{4}+x^{3}+x^{2}+x+1
$$

Then, its invariants are

$$
\begin{aligned}
& \tilde{J}_{2}=\frac{9}{5}, \tilde{J}_{3}=\frac{81}{2450}, \tilde{J}_{4}=\frac{837}{1568}, \tilde{J}_{5}=\frac{2187}{109760}, \tilde{J}_{6}=-\frac{6885}{43904} \\
& \tilde{J}_{7}=-\frac{3645}{1229312}, \tilde{J}_{8}=-\frac{410427}{17210368}, \tilde{J}_{9}=\frac{234009}{172103680}, \tilde{J}_{10}=\frac{5972697}{860518400}
\end{aligned}
$$

Then evaluating all expressions as in Shioda's paper we have

$$
\begin{aligned}
& A_{6}==-\frac{3645}{9604}, \quad A_{7}=\frac{130491}{439040}, \\
& B_{7}=\frac{130491}{351232}, \quad B_{9}=\frac{1414989}{172103680}, \\
& C_{9}=\frac{809753208633}{376476800000}, \quad C_{10}=-\frac{51828148570131}{150590720000}, \\
& D_{10}=-\frac{19194738471171}{385512243200}, \quad A_{16}=-\frac{1097050897751848407621}{925614895923200000} . \\
& B_{8}=\frac{143437311}{21512960}
\end{aligned}
$$


Substituting all these values in the first equation of [23. Thm. 5] we get the value

$$
-\frac{546607935510034107123}{462807447961600000} \neq 0 \text {. }
$$

This implies that the relations determined by Shioda are not correct.

Indeed, if you take any random binary octavics then its invariants will not satisfy the Shioda's relations. Since the results in 23] do not hold, then one needs to determine explicitly the algebraic relations between the invariants in order to have an explicit description of the ring of invariants $\mathcal{R}_{8}$ and its field of fractions $\mathcal{S}_{8}$. This will be our goal for the rest of this paper.

3.2.2. Algebraic dependencies among the invariants. In this section we will determine algebraic relations among the invariants $J_{2}, \ldots, J_{8}$. We will use computational algebra techniques such as elimination by resultants, Groebner bases, etc. Any computer algebra package can be used to reproduce our results. Once obtained, these results can be easily verified. All our results are organized in a Maple package and will be freely made available.

Without loss of generality we can assume that the generic binary octavic is given by

$$
\begin{aligned}
f(X, 1) & =X(X-1)\left(X^{5}-s_{1} X^{4}+s_{2} X^{3}-s_{3} X^{2}+s_{4} X-s_{5}\right) \\
& =X^{7}-\left(s_{1}+1\right) X^{6}+\left(s_{2}+s_{1}\right) X^{5}-\left(s_{3}+s_{2}\right) X^{4}+\left(s_{4}+s_{3}\right) X^{3}-\left(s_{4}+s_{5}\right) X^{2}+s_{5} X
\end{aligned}
$$

Denote by

$$
a:=s_{1}+s_{2}, b:=s_{2}+s_{3}, c:=s_{3}+s_{4}, d:=s_{4}+s_{5}, s:=s_{5} .
$$

Then we have

$$
f(X, 1)=X^{7}+(-1+b-s+d-c-a) X^{6}+a X^{5}-b X^{4}+c X^{3}-d X^{2}+s X
$$

We first compute the $J_{2}, \ldots, J_{10}$ for $f(X, 1)$.

$$
\begin{aligned}
J_{2}(f)= & -35 s+10 d-10 d b+10 d s-10 d^{2}+10 d c+10 d a-5 a c+2 b^{2} \\
J_{3}(f)= & -75 c^{2}+75 c^{2} b-75 c^{2} s+75 c^{2} d-75 c^{3}-75 c^{2} a-75 d a^{2}-12 b^{3}+110 d b-110 d b^{2}+110 d b s-110 d^{2} b \\
& +110 d b c+110 d b a+175 a s-175 a s b+175 a s^{2}-175 a s d+175 a s c+175 a^{2} s-735 b s+175 d c+45 c b a,
\end{aligned}
$$

$J_{4}, \ldots, J_{8}$ are largeer expressions and we do not display them.

Our goal is to express $J_{8}, J_{9}, J_{10}$ in terms of $j_{2}, \ldots, J_{7}$. Indeed, from Thm. 5 it is enough to express $J_{8}$ in terms of $J_{2}, \ldots, J_{7}$. Since in [23] the syzygies include expressing $J_{9}$ and $J_{10}$ in terms of $J_{2}, \ldots, J_{7}$ we will comment on how that can be done also.

We have the following system of equations

$$
\left\{\begin{array}{l}
F_{2}:=J_{2}-J_{2}(a, b, c, d, s)=0 \\
F_{3}:=J_{3}-J_{3}(a, b, c, d, s)=0 \\
F_{4}:=J_{4}-J_{4}(a, b, c, d, s)=0 \\
F_{5}:=J_{5}-J_{5}(a, b, c, d, s)=0 \\
F_{6}:=J_{6}-J_{6}(a, b, c, d, s)=0 \\
F_{7}:=J_{7}-J_{7}(a, b, c, d, s)=0 \\
F_{8}:=J_{8}-J_{8}(a, b, c, d, s)=0
\end{array}\right.
$$

We compute the equation of $J_{8}$ in terms of $J_{2}, \ldots, J_{7}$ using the following technique. Take the resultant with respect to $a$ of the polynomials $F_{i}, F_{8}$, for $i=2, \ldots 7$. Let $G_{i}:=\operatorname{Res}\left(F_{i}, F_{8}, a\right)$, for $i=2, \ldots 7$. For each resultant we want to factor the result and take the primitive part. It is exactly this part that is important and it is not usually done by implementations of Grobener basis algorithms. In many cases the resultant will be factored to a power or will have factors which imply that $J_{14}=0$. Since we are computing in an integral domain, we cancel such factors.

We continue now with the system $G_{i}:=\operatorname{Res}\left(F_{i}, F_{8}, a\right)$, for $i=2, \ldots 7$ and compute the resultants $H_{i}:=$ $\operatorname{Res}\left(G_{i}, G_{7}, b\right)$ for $i=2, \ldots 6$. Hence, we are left 5 equations and transcendentals $c, d, s$. Continuing this process we get a degree 8 equation of $J_{8}$ in terms of the other $J_{2}, \ldots, J_{7}$, as expected by Shioda; see [23, pg. 1044]. Its leading 
monomial has coefficient $2^{2} \cdot 3^{20} \cdot 5^{12}$. Since we are assuming that the characteristic of the field is $\neq 2,3,5,7$ then we can divide by this coefficient. Hence, denote the minimal quintic by

$$
J_{8}^{5}+c_{4} J_{8}^{4}+\cdots+c_{1} J_{8}+c_{0}=0 .
$$

Since this equation is a homogenous equation of degree $40 \mathrm{in} J_{2}, \ldots, J_{8}$, then all other coefficients of $J_{8}$ are homogenous polynomials in $J_{2}, \ldots, J_{7}$ of degree $8,16,24,32,40$ respectively. We denote the primitive part of each of these coefficients by $I_{8}, I_{16}, I_{24}, I_{32}, I_{40}$.

For now on we use the following notation

$$
J_{2}:=a, \quad J_{3}:=b, \quad J_{4}:=c, \quad J_{5}:=d, \quad J_{6}:=e, \quad J_{7}:=f,
$$

to display the expressions of $I_{8}, I_{16}, I_{24}, I_{32}, I_{40}$.

$$
\begin{aligned}
I_{8} & =-2^{7} 7^{5} a^{4}+2^{2} 5^{3} 7^{3} 3 a^{2} c+2^{6} 3^{3} 7^{2} a b^{2}+2^{3} 5^{4} 7^{2} a e-2^{2} 3^{5} 5^{2} 7 b d-3^{3} 5^{4} 17 c^{2} \\
I_{16} & =2^{2} 3^{7} 5^{5} 7^{5} a^{3} d^{2}+2^{2} 3^{8} 5^{6} 7 \cdot 11 b c^{2} d+2^{3} 5^{8} 7^{4} a^{2} e^{2}-2 \cdot 3^{7} 5^{6} 7^{3} a b c f+2^{9} 3^{6} 7^{4} a^{2} b^{4}-3 \cdot 2^{3} 5^{5} 7^{6} \cdot 31 a^{3} c e-2^{2} 3^{8} 5^{6} 7^{3} a c d^{2} \\
& +2^{3} 3^{4} 5^{5} 7^{2} 11^{2} b^{2} c e-2 \cdot 3^{3} 5^{7} 7^{3} 13 a^{2} c^{3}-57173^{5} 5^{4} 7^{2} a b^{2} c^{2}+2^{6} 3^{6} 5^{3} 7^{5} a^{3} b f+2^{2} 3^{8} 5^{5} 7^{4} a^{2} d f+2^{7} 3^{5} 5^{2} 7^{6} a^{4} b d \\
& -3^{8} 5^{7} 7^{3} a f^{2}-2^{3} 3^{10} 5^{4} 7^{2} b^{2} d^{2}-2^{5} 3^{7} 5^{2} 7^{2} 19 b^{4} c-2^{2} 3^{7} 5^{7} 7^{2} d^{2} e+2^{11} 7^{10} a^{8}+2^{6} 3^{4} 5^{2} 7^{5} 43 a^{3} b^{2} c-2^{8} 5^{4} 7^{7} a^{5} e \\
& -2^{2} 3^{6} 5^{5} 7^{4} 11 a^{2} b c d+2^{7} 3^{3} 5^{4} 7^{4} a^{2} b^{2} e+2^{3} 3^{3} 5^{4} 7^{5} 491 a^{4} c^{2}-2^{6} 3^{8} 5^{2} 7^{3} a b^{3} d-2^{5} 3^{9} 5^{3} 7^{2} b^{3} f-2^{3} 3^{5} 5^{6} 7^{3} a b d e \\
& -2^{2} 3^{6} 5^{7} 7^{2} b e f-2^{11} 3^{3} 7^{7} a^{5} b^{2}+2^{2} 3^{2} 5^{7} 7^{2} 601 a c^{2} e-2^{10} 3^{2} 5^{2} 7^{8} a^{6} c+3^{5} 5^{9} 19 c^{4}+2^{2} 3^{9} 5^{7} 7^{2} 17 c d f-2 \cdot 5^{9} 7^{2} c e^{2}
\end{aligned}
$$

The invariant $I_{32}$ is an equation of degree $14,8,8,4,5,4$ in $a, b, c, d, e, f$ respectively. We denote it as $I_{32}=$ $\sum_{i=0}^{14} b_{i} J_{2}$ and display its coefficients as follows:

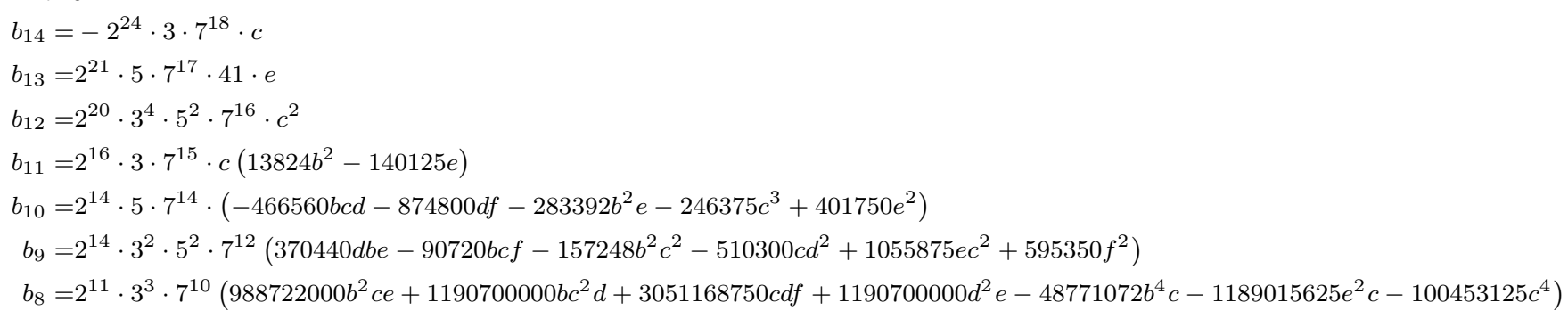

Next we describe $I_{40}$. It is a degree 17 polynomial in $J_{2}$ and we denote it by $I_{40}=\sum_{i_{0}}^{17} A_{i} J_{2}^{i}$. Then, we have

$$
\begin{aligned}
A_{17} & =2^{33} \cdot 7^{22} \\
A_{16} & =2^{28} \cdot 3^{2} \cdot 5 \cdot 7^{21} \cdot c^{2} \\
A_{15} & =2^{28} \cdot 3 \cdot 5^{4} \cdot 7^{20} \cdot c e \\
A_{14} & =2^{22} \cdot 5 \cdot 7^{18}\left(193536 b^{2} e-223425 c^{3}-266875 e^{2}\right) \\
A_{13} & =2^{24} \cdot 3^{4} \cdot 5^{2} \cdot 7^{17}\left(-168 b^{2} c^{2}-1975 c^{2} e-1890 f^{2}-1680 b d e+504 b c f\right) \\
A_{12} & =2^{18} \cdot 3 \cdot 5^{3} \cdot 7^{16}\left(489880 c d f-6531840 d^{2} e+2668750 c e^{2}+924075 c^{4}+326592 b c^{2} d-1935360 b^{2} c e\right) \\
A_{11} & =2^{19} \cdot 5 \cdot 7^{15}\left(1020600000 b c d e+96519600 b^{2} c^{3}-172226250 c^{2} d^{2}-41803776 b^{4} e+143184375 c^{3} e\right. \\
& \left.+115290000 b^{2} e^{2}+602791875 f^{2} c-71225000 e^{3}-306180000 b c^{2} f+1262992500 d e f\right)
\end{aligned}
$$

The other coefficients are displayed in the Appendix 3.5

Problem 1. Express all invariants $I_{8}, I_{16}, I_{24}, I_{32}, I_{40}$ in terms of the transvectants of the binary octavics.

We summerize the above in the following theorem.

Theorem 6. The invariants $J_{2}, \ldots, J_{8}$ satisfy the following equation

$$
J_{8}^{5}+\frac{I_{8}}{3^{4} \cdot 5^{3}} J_{8}^{4}+2 \cdot \frac{I_{16}}{3^{8} \cdot 5^{6}} J_{8}^{3}+\frac{I_{24}}{2 \cdot 3^{12} \cdot 5^{6}} J_{8}^{2}+\frac{I_{32}}{3^{16} \cdot 5^{10}} J_{8}+\frac{I_{40}}{2^{2} \cdot 3^{20} \cdot 5^{12}}=0,
$$

Proof. To prove that this relation holds we take a generic octavic

$$
f(x, z)=\sum_{i=1}^{8} a_{i} x^{i} z^{8-i} .
$$


Compute the invariants $J_{2}, \ldots, J_{8}$ and substitute them in the Eq. (16). We see that the equation is satisfied. This completes the proof.

Remark 4. i) In terms of the coefficients of the binary octavic the above equation is a degree 40 homogenous equation.

ii) The equation has degrees in $J_{i}, i=2, \ldots, 8$, respectively $17,10,10,6,6,5,5$.

iii) Similar relations as that in previous Theorem can be determined for $J_{9}$ and $J_{10}$ in terms of $J_{2}, \ldots, J_{7}$. However, such relations, as expected, are very large to display.

iv) In $[23$ it is commented that the field of fractions of $\mathcal{R}$ is determined by a degree 5 equation

$$
J_{8}^{5}+a_{1} J_{8}^{4}+\ldots \alpha_{5}=0,
$$

where $a_{1}, \ldots, a_{5}$ are homogenous elements in $\mathbb{Q}\left[J_{2}, \ldots, J_{7}\right]$ but are not computed; see page 1043. That equation is precisely Eq. (16).

v) All coefficients of these equation can be expressed in terms of the transvectants of binary octavics.

vi) The reader can check the correctness of the above equation in [19]

Lemma 5. The following hold true for any octavic.

i) If an octavic has a root of multiplicity exactly four then

$$
\begin{aligned}
I_{8} & =2^{11} \cdot 3^{6} \cdot 5^{4} \cdot r^{8}, \quad I_{16}=2^{22} \cdot 3^{12} \cdot 5^{7} r^{16}, \quad I_{24}=2^{35} \cdot 3^{18} \cdot 5^{7} r^{24}, \\
I_{32} & =2^{44} \cdot 3^{24} \cdot 5^{11} r^{32}, \quad I_{40}=2^{57} \cdot 3^{30} \cdot 5^{12} r^{40},
\end{aligned}
$$

for some $r \neq 0$. Moreover, if the octavic has equation

$$
f(x, y)=x^{4}\left(a x^{4}+b x^{3} y+c x^{2} y^{2}+d x y^{3}+e y^{4}\right),
$$

then $r=e$.

ii) If an octavic has a root of multiplicity 5 then

$$
I_{8 i}=0, \text { for } i=1, \ldots, 5 .
$$

Proof. i) The proof follows Theorem 4 part i) or by direct computation.

ii) Since all $I_{8 i}$ for $i=1, \ldots, 5$ are all homogenous polynomials in terms of $J_{2}, \ldots, J_{7}$ then this is an immediate consequence of Theorem 4

Corollary 2. If $J_{4}=J_{5}=J_{6}=J_{7}=0$, then $I_{24}=I_{32}=I_{40}=0$. In this case, the Eq. (16) becomes

$$
J_{8}\left(-10125 J_{8}+1075648 J_{2}^{4}-42336 J_{3}^{2} J_{2}\right)=0
$$

The equation Eq. (16) corrects the result of [23, Thm. 5]. To compute the other syzygies we follow a similar technique replacing $J_{8}$ by $J_{9}$ or $J_{10}$. Indeed, both such cases are a bit easier from the computational point of view. For our purposes of determining the field of invariants $\mathcal{S}_{8}$ the Eq. (16) is enough.

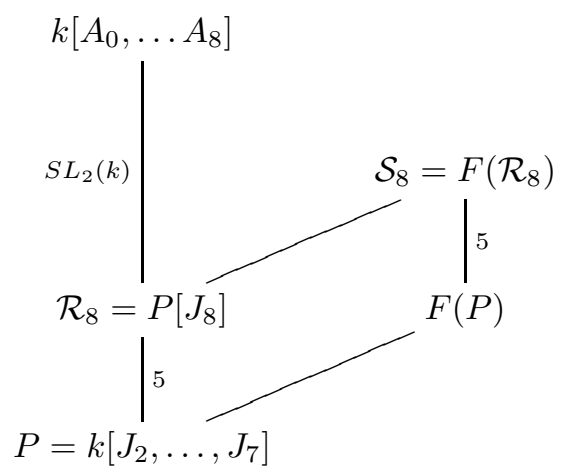

Figure 1. The ring of invariants $\mathcal{R}_{8}$ and its field of fractions 
Example 2. Let $C$ be the generic genus 3 hyperelliptic curve with automorphism group $\operatorname{Aut}(C) \cong \mathbb{Z}_{2} \times D_{8}$. Then $C$ has equation

$$
C: y^{2}=x^{8}+\lambda x^{4}+1
$$

Then, invariants $I_{8 i}, i=1, \ldots 5$ of the corresponding binary form are given below.

$$
\begin{aligned}
I_{8} & =-2^{11} \cdot 3^{4} \cdot 5^{4}\left(9 \lambda^{2}-2450\right)(\lambda-14)^{3}(\lambda+14)^{3} \\
I_{16} & =2^{22} \cdot 3^{9} \cdot 5^{7}\left(9 \lambda^{2}+980\right)\left(3 \lambda^{2}-1960\right)(\lambda-14)^{6}(\lambda+14)^{6} \\
I_{24} & =-2^{35} \cdot 3^{12} \cdot 5^{7}\left(9 \lambda^{2}-9310\right)\left(9 \lambda^{2}+980\right)^{2}(\lambda-14)^{9}(\lambda+14)^{9} \\
I_{32} & =2^{44} \cdot 3^{16} \cdot 5^{11}\left(9 \lambda^{2}-12740\right)\left(9 \lambda^{2}+980\right)^{3}(\lambda-14)^{12}(\lambda+14)^{12} \\
I_{40} & =-2^{57} \cdot 3^{21} \cdot 5^{12}\left(3 \lambda^{2}-5390\right)\left(9 \lambda^{2}+980\right)^{4}(\lambda-14)^{15}(\lambda+14)^{15}
\end{aligned}
$$

If $\lambda^{2}=14$ then $I_{8 i}=0$, for $i=1, \ldots, 5$ dhe $J_{i}=0$ for $i=4, \ldots, 8$. This corresponds to the single curve with automorphism group $\operatorname{Aut}(C) \cong \mathbb{Z}_{2} \times S_{4}$.

Theorem 7. Two genus 3 hyperelliptic curves $C$ and $C^{\prime}$ in Weierstrass form, given by equations

$$
C: Z^{2}=f(X, Y) \text { and } C^{\prime}: z^{2}=g(X, Y)
$$

are isomorphic over $k$ if and only if there exists some $\lambda \in k \backslash\{0\}$ such that

$$
J_{i}(C)=\lambda^{i} J_{i}\left(C^{\prime}\right), \text { for } i=2, \ldots, 7,
$$

and $J_{2}, \ldots J_{8}$ satisfy the Eq. (16). Moreover, the automorphism is given by

$$
\begin{gathered}
C \rightarrow C^{\prime} \\
{\left[\begin{array}{l}
X \\
Y
\end{array}\right] \rightarrow M \cdot\left[\begin{array}{l}
X \\
Y
\end{array}\right]}
\end{gathered}
$$

where $M \in G L_{2}(k)$ and $\lambda=(\operatorname{det} M)^{4}$.

Proof. The proof follows directly from the properties of invariants and Lemma 3 ,

The above theorem gives a necessary and suffiecient condition for two hyperelliptic curves to be isomorphic. However, $G L(2, k)$-invariants are prefered for identifying the isomorphism classes of curves. In order to find such invariants we need to determine the field of fractions of $\mathcal{R}_{8}=k\left[J_{2}, \ldots, J_{7}, J_{8}\right]$.

3.3. On the invariant field of $G L_{2}(k)$. Let us assume that $J_{2}, J_{3}, J_{4}, J_{5}$ are all nonzero. Define the invariants

$$
t_{1}:=\frac{J_{3}^{2}}{J_{2}^{3}}, \quad t_{2}:=\frac{J_{4}}{J_{2}^{2}}, \quad t_{3}:=\frac{J_{5}}{J_{2} \cdot J_{3}}, \quad t_{4}:=\frac{J_{6}}{J_{2} \cdot J_{4}}, \quad t_{5}:=\frac{J_{7}}{J_{2} \cdot J_{5}}, \quad t_{6}:=\frac{J_{8}}{J_{2}^{4}} .
$$

Such invariants have the same degree in numerator and denumerator, therefore they are $G L(2, k)$-invariants. Hence, $t_{1}, \ldots, t_{6} \in \mathcal{S}_{8}$. For analogy with the genus 2 case, we call them absolute invariants. For any two isomorphic genus 3 hyperelliptic curves $C$ and $C^{\prime}$ we have $t_{j}(C)=t_{j}\left(C^{\prime}\right)$, for $j=1, \ldots, 6$. We would prefer an if and only if statement.

By substituting in Eq.(16) get an affine equation of the hyperelliptic moduli of genus 3 as

$$
T\left(t_{1}, \ldots, t_{6}\right)=0
$$

This is an algebraic variety of dimension 5 . It has degrees in $t_{1}, \ldots, t_{6}$ respectively as $5,10,6,6,5,5$ and it has 25464 terms. We denote this variety by $\mathcal{T}_{3}$. The equation of $\mathcal{T}_{3}$ is explicitely computed and very useful in the arithmetic of genus 3 curves. The reader can check it at [19].

Then we have the following theorem.

Theorem 8. The field of invariants of binary octavics is $\mathcal{S}_{8}=k\left(t_{1}, \ldots, t_{6}\right)$, where $t_{1}, \ldots, t_{6}$ satisfy the equation (19). 
Proof. The proof of the theorem follows directly from Thm. ?? in Shioda. However, since that is based on Thm. 5 which contains syzygies which are incorrect, we provide a direct proof for this result.

We denote the roots of the octavic $f(X, Y)$ by $\left(\alpha_{i}, \beta_{i}\right)$. Every $g \in G L_{2}(k)$ which fixes $f(X, Y)$ permutes these roots. Thus there is an $S_{8}$ action on $\left\{\alpha_{0}, \ldots, \alpha_{7}\right\}$. The fixed field is the invariant field of $G L_{2}(k)$ which we denote by $\mathcal{S}_{8}$. We can fix $\alpha_{5}=0, \alpha_{6}=1$, and $\alpha_{7}=\infty$. Let $s_{1}, \ldots, s_{5}$ denote the symmetric polynomials of $\alpha_{0}, \ldots, \alpha_{4}$. Then $\left[k\left(\alpha_{0}, \ldots, \alpha_{4}\right): k\left(s_{1}, \ldots, s_{5}\right)\right]=120$. Thus, $\left[k\left(s_{1}, \ldots, s_{5}\right): \mathcal{S}_{8}\right]=6 \cdot 7 \cdot 8=336$.

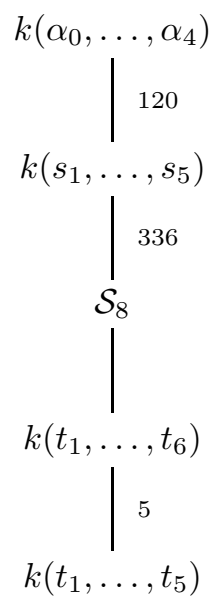

Our goal is to determine $\mathcal{S}_{8}$.

Since $t_{1}, \ldots, t_{6}$ are $G L(2, k)$-invariants then $k\left(t_{1}, \ldots, t_{6}\right) \subset \mathcal{S}_{8}$. We know that $\left[k\left(t_{1}, \ldots, t_{6}\right): k\left(t_{1}, \ldots, t_{5}\right)=5\right.$, since the degree in $t_{6}$ of the irreducible polynomial from Eq. (19) is 5 . If we show that $\left[k\left(s_{1}, \ldots, s_{5}\right): k\left(t_{1}, \ldots, t_{5}\right)\right]=$ $5 \cdot 336$, or equivalently $\left[k\left(s_{1}, \ldots, s_{5}\right): k\left(t_{1}, \ldots, t_{6}\right)\right]=336$ then we are done.

The proof is computational. Compute $t_{1}, \ldots, t_{5}$ in terms of $s_{1}, \ldots, s_{5}$. This is computationally easy and we do not display these expressions here. By Bezout's theorem we know that the degree $d=\left[k\left(s_{1}, \ldots, s_{5}\right): k\left(t_{1}, \ldots, t_{5}\right)\right]$ is $d \leq 6 \cdot 4 \cdot 5 \cdot 6 \cdot 7$, becaus ethe degrees of $i_{1}, \ldots, i_{5}$ are respectively $6,4,5,6,7$. There is at least one more solution at infinity. Moreover, $d$ must be divisible by $5 \cdot 336$. Hence, $d=5 \cdot 336$ or $d=2 \cdot 5 \cdot 336$.

From the system of equations we eliminate first $s_{5}$. Continuing via the resultants we eliminate also $s_{1}$ and $s_{4}$. We are left with two equations of degree 36 and 56. From Bezout's theorem, the degree $d \leq 36 \cdot 56$ and divisible by 1680 . Hence $d=1680$ and the proof is complete.

Corollary 3. Two hyperelliptic genus 3 curves with nonzero invariants $J_{2}, J_{3}, J_{4}, J_{5}$ are isomorphic if and only if they correspond to the same point on the algebraic variety $\mathcal{T}_{3}$.

Proof. The proof is an imediate consequence of the previous Theorem.

Since the moduli space of hyperelliptic curves is a rational variety then $\mathcal{T}_{3}$ must have a birational parametrization. Finding such parametrization via an equation of this size is very difficult.

3.4. Cases when $t_{1}, \ldots, t_{6}$ are not defined. To describe the moduli points in cases when absolute invariants are not defined is not difficult. In this case, one has to treat each case separately when any of the invariants $J_{2}, \ldots J_{5}$ are zero.

Indeed, we can define invariants depending of which of the invariants is nonzero. If $J_{2} \neq 0$, then we define

$$
i_{1}=\frac{J_{3}^{2}}{J_{2}^{3}}, \quad i_{2}=\frac{J_{4}}{J_{2}^{2}}, \quad i_{3}=\frac{J_{5}^{2}}{J_{2}^{5}}, \quad i_{4}=\frac{J_{6}}{J_{2}^{3}}, \quad i_{5}=\frac{J_{7}^{2}}{J_{2}^{7}}, \quad i_{6}=\frac{J_{8}}{J_{2}^{4}}
$$

If $J_{2}=0$ then we pick the smallest degree invariant among $J_{3}, \ldots, J_{7}$ which is not zero. This is possible because if $J_{2}=\cdots=J_{7}=0$, then from Lemma ?? the binary octavic has a double root, hence we don't have a genus 3 curve. For example, if $J_{3} \neq 0$, then we define

$$
i_{1}=\frac{J_{2}^{3}}{J_{3}^{2}}, \quad i_{2}=\frac{J_{4}}{J_{3} \cdot J_{4}}, \quad i_{3}=\frac{J_{8}}{J_{3} \cdot J_{5}}, \quad i_{4}=\frac{J_{4}^{3}}{J_{3}^{4}}, \quad i_{5}=\frac{J_{5}^{3}}{J_{3}^{5}},
$$


Such invariants have high degree in some cases and therefore are not suitable for computations. Hence, we prefer invariants $t_{1}, \ldots, t_{6}$ defined in Eq. (18).

In the next example we see what happens in the case when all $J_{2}=J_{3}=J_{4}=J_{5}=0$. We see that we get a genus 0 curve in the hyperelliptic moduli $\mathcal{H}_{3}$.

Example 3. Let us assume that $J_{2}=J_{3}=J_{4}=J_{5}=0$. In this case $I_{8}$ and $I_{8}=I_{16}=0$ and

$$
I_{24}-2679687500000 u^{4}, \quad I_{32}-3204948120117187500 u^{3} v^{2}, \quad I_{40}=-306653442317962646484375 u^{2} v^{4}
$$

where $u, v \in k[a, b, c, d, e]$. Moreover,

$$
\left(25 I_{24} I_{40}-2 I_{32}^{2}\right)\left(25 I_{24} I_{40}+2 I_{32}^{2}\right)=0
$$

In this case, the Eq. (16) becomes

$$
2125764 J_{8}^{5}-343000000 J_{6}^{4} J_{8}^{2}+16206750000 J_{6}^{3} J_{7}^{2} J_{8}-191442234375 J_{6}^{2} J_{7}^{4}=0
$$

By defining

the Eq. (16) becomes

$$
\tau_{1}:=\frac{J_{7}^{6}}{J_{6}^{7}}, \quad \tau_{2}=\frac{J_{8}^{3}}{J_{6}^{4}}
$$

$$
\begin{array}{r}
-1064211156161261718750000000000 \tau_{1} \tau_{2}+40353607000000000000000000 \tau_{2}{ }^{2}+4649919888623184000000 \tau_{2}{ }^{4} \\
-9606056659007943744 \tau_{2}{ }^{5}-750282026508000000000000 \tau_{2}{ }^{3}+7016382605513364494808197021484375 \tau_{1}{ }^{2} \\
-19786546042268119734375000000 \tau_{1} \tau_{2}{ }^{2}=0
\end{array}
$$

This is a genus 0 curve that can be parametrized as follows

$$
\tau_{1}=2^{46} 3^{6} 5^{60} 7^{36} \cdot \frac{\left(t+2^{11} 3^{9} 5^{18} 7^{12}\right)^{2}}{t^{5}}, \quad \tau_{2}=\frac{2^{4} 5^{6} 7^{3}}{3^{12}} \cdot \frac{\left(t+2^{11} 3^{9} 5^{18} 7^{12}\right)^{2}}{t^{2}}
$$

It is possible in this case to express the equation of the curve in terms of the parameter $t$.

3.5. A computational package for genus 3 hyperelliptic curves. All the computational results described in this paper are implemented in a Maple package which will be made freely available. This package among other things computes the following:

i) Invariants $J_{i}$ for $i=1, \ldots, 10$. Their formulas are given in terms of the coefficients of a generic octavic

$$
f(X, Y)=\sum_{i=1}^{8} a_{i} X^{i} Y^{8-i}
$$

and can be evaluated on any given octavic.

ii) Invariants $I_{5 i}$, for $i=1, \ldots, 5$. Their formulas are given in terms of $J_{2}, \ldots, J_{7}$ and can be evaluated on any octavic.

iii) The equation (16) in terms of the invariants $J_{2}, \ldots, J_{8}$.

iv) The equation (19) in terms of invariants $i_{1}, \ldots, i_{6}$.

Some problems which we are further studying are: finding a minimal model of a genus 3 curve over its minimal field of definition, determining an algorithm which determines when the field of moduli is a field of definition, and describing the loci of curves with fixed automorphism group in terms of invariants $t_{1}, \ldots, t_{6}$. For these problems and other computational aspects of genus 3 hyperelliptic curves see [21].

\section{REFERENCES}

[1] R. Alagna, Le relazioni fra gl'invarianti d'una forma qualunque d'ottavo ordine, Rendiconti del Circolo Matematico di Palermo 6 (1892), no. 1, 77-99 (Italian).

[2] Le relazioni fra gl'invarianti d'una forma qualunque d'ottavo ordine, Rendiconti del Circolo Matematico di Palermo 10 (1896), no. 1, 41-74 (Italian).

[3] W. D. Geyer, Invarianten binärer Formen, Classification of algebraic varieties and compact complex manifolds, Springer, Berlin, 1974, pp. 36-69. Lecture Notes in Math., Vol. 412 (German). MR0374142 (51 \#10342)

[4] J. H. Grace and A. Young, The algebra of invariants, Cambridge Library Collection, Cambridge University Press, Cambridge, 2010. Reprint of the 1903 original. 
[5] J. Gutierrez, D. Sevilla, and T. Shaska, Hyperelliptic curves of genus 3 with prescribed automorphism group, Computational aspects of algebraic curves, Lecture Notes Ser. Comput. vol. 13, World Sci. Publ., Hackensack, NJ, 2005, pp. 109-123.

[6] J. Gutierrez and T. Shaska, Hyperelliptic curves with extra involutions, LMS J. Comput. Math. 8 (2005), 102-115 (electronic).

[7] W. Haboush, Reductive groups are geometrically reductive, Ann. of Math. 102 (1975), 68-83.

[8] D. Hilbert, Theory of algebraic invariants, Cambridge University Press, Cambridge, 1993. Translated from the German and with a preface by Reinhard C. Laubenbacher; Edited and with an introduction by Bernd Sturmfels.

[9] Jun-ichi Igusa, Arithmetic variety of moduli for genus two, Ann. of Math. (2) $\mathbf{7 2}$ (1960), 612-649.

[10] P. Katsylo, Rationality of the moduli variety of curves of genus 3, Comment. Math. Helv. 71 (1996), no. 4, $507-524$.

[11] V. Krishnamoorthy, T. Shaska, and H. Völklein, Invariants of binary forms, Progress in Galois theory, Dev. Math. vol. 12, Springer, New York, 2005, pp. 101-122.

[12] M. Nagata, Invariants of a group in an affine ring, J. Math, Kyoto Univ. 3 (1964), 369-377.

[13] T. Shaska, Some special families of hyperelliptic curves, J. Algebra Appl. 3 (2004), no. 1, 75-89.

[14] _ Computational aspects of hyperelliptic curves, Computer mathematics, Lecture Notes Ser. Comput. vol. 10, World Sci. Publ., River Edge, NJ, 2003, pp. 248-257.

[15] Determining the automorphism group of a hyperelliptic curve, Proceedings of the 2003 International Symposium on Symbolic and Algebraic Computation, ACM, New York, 2003, pp. 248-254 (electronic).

[16] _ Some open problems in computational algebraic geometry, Albanian J. Math. 1 (2007), no. 4, $297-319$.

[17] _ Genus two curves covering elliptic curves: a computational approach, Computational aspects of algebraic curves, Lecture Notes Ser. Comput. vol. 13, World Sci. Publ., Hackensack, NJ, 2005, pp. 206-231.

[18] _ Families of genus 2 curves with many elliptic subcovers, to appear.

[19] _ Algebraic Curves (2012), https://sites.google.com/a/oakland.edu/algebra/algebraic-curves

[20] T. Shaska and J. L. Thompson, On the generic curve of genus 3, Affine algebraic geometry, Contemp. Math. vol. 369, Amer. Math. Soc. Providence, RI, 2005, pp. 233-243.

[21] T. Shaska and F. Thompson, Genus 3 hyperelliptic curves with automorphisms and their minimal equations, to appear.

[22] T. Shaska and H. Völklein, Elliptic subfields and automorphisms of genus 2 function fields, Algebra, arithmetic and geometry with applications (West Lafayette, IN, 2000), Springer, Berlin, 2004, pp. 703-723.

[23] Tetsuji Shioda, On the graded ring of invariants of binary octavics, Amer. J. Math. 89 (1967), 1022-1046.

[24] Von Gall, Ueber das vollständige System einer binären Form achter Ordnung, Math. Ann. 17 (1880), no. 1, 139-152 (German).

[25] _ Das vollständige Formensystem einer binären Form achter Ordnung, Math. Ann. 17 (1880), no. 1, 31-51 (German). 


\section{THE ALGEBRAIC RELATIONS AMONG INVARIANTS}

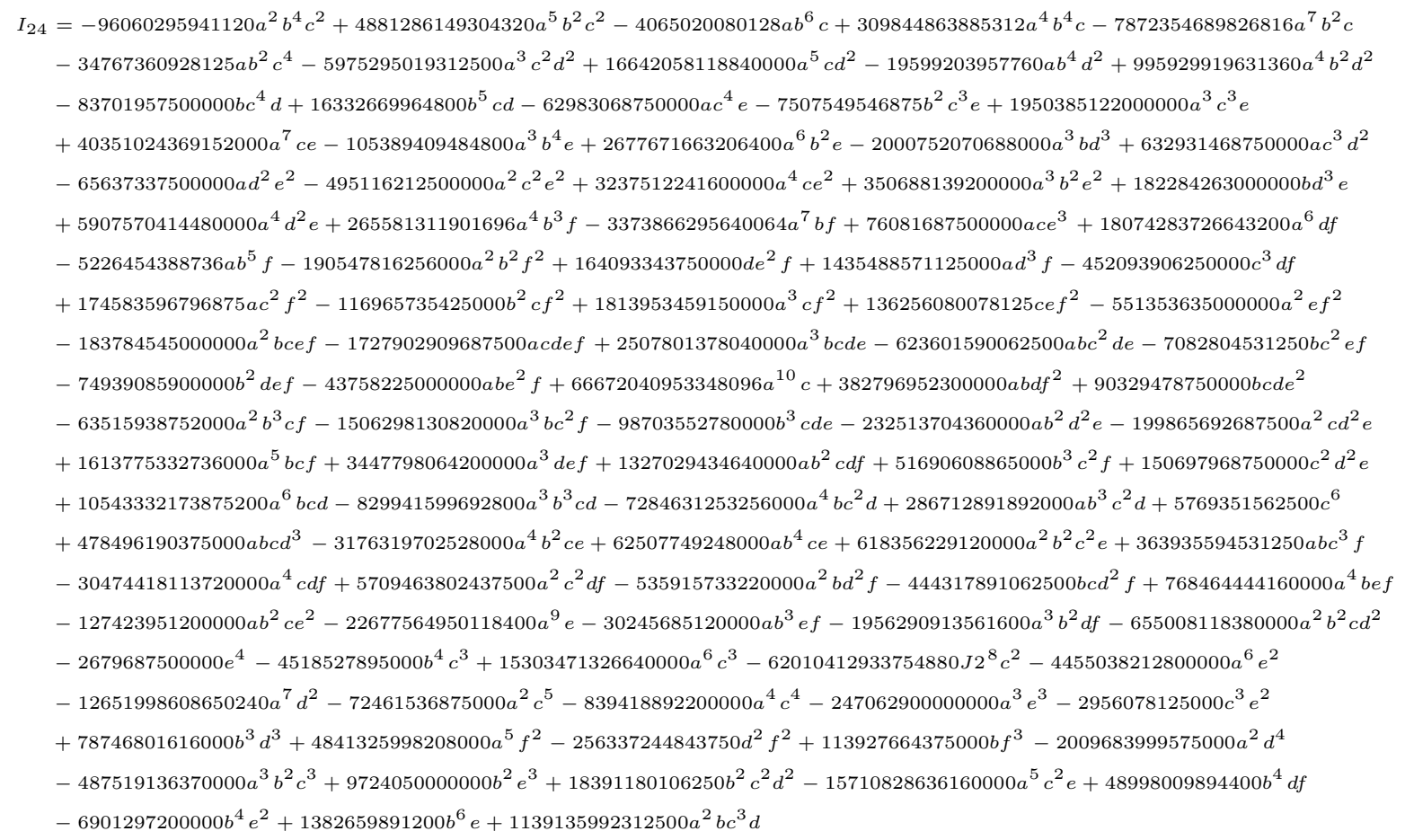

$b_{7}=2^{8} \cdot 3 \cdot 5 \cdot 7^{10}\left(2821754880 b^{3} c d-23346225000 d e f+7031205000 b c^{2} f+5290790400 f d b^{2}+469476000 b^{2} c^{3}+19190925000 c^{2} d^{2}\right)$

$\left.-7589671875 c^{3} e+856977408 b^{4} e-2429784000 b^{2} e^{2}-27938925000 f^{2} c+1599500000 e^{3}-20182365000 b c d e\right)$

$b_{6}=2^{6} \cdot 3^{5} \cdot 5^{2} \cdot 7^{8}\left(+1590540000 d b e^{2}-1793022000 b^{2} c^{2} e-995742720 d b^{3} e-8574693750 c d^{2} e-1574370000 b c^{3} d-2601112500 c^{2} d f\right.$

$-3429216000 b d^{2} f+243855360 b^{3} c f-1371686400 b^{2} f^{2}+2976750000 e f^{2}+130056192 b^{4} c^{2}+2192312500 e^{2} c^{2}+276921875 c^{5}$

-1199520000 bcef)

$b_{5}=2^{5} \cdot 3 \cdot 7^{7}\left(91423434375000 b c^{2} d e-7688302272000 b^{4} c e-18517766400000 d^{2} b^{2} e-18517766400000 b^{3} c^{2} d-64531023046875 c^{3} d^{2}\right.$

$-52081218000000 b c d^{3}+18491571000000 b^{2} c e^{2}-36095751562500 b c^{3} f+26040609000000 f^{2} d b-97652283750000 f d^{3}$

$+2775093750000 e c^{4}+46632649218750 d^{2} e^{2}-10697312500000 e^{3} c+81089859375000 c^{2} f^{2}+143497954687500 c d e f$

$\left.+252829237248 b^{6} c+3794809500000 b^{2} c^{4}-47451776400000 b^{2} c d f\right)$

$b_{4}=2^{3} \cdot 5 \cdot 7^{5}\left(2563321813920000 b^{3} c d e-2551979682000000 b f^{3}+11278838773125000 d^{2} f^{2}+93467500000000 e^{4}-335986353561600 b^{4} d f\right.$

$-36280995545088 b^{6} e+154301003136000 b^{4} e^{2}-203149296000000 b^{2} e^{3}-1549416235500000 d^{3} b e-5506503778125000 d e^{2} f$

$-63420152343750 c^{6}-673882453125000 c^{3} e^{2}-1958571267187500 c e f^{2}+290775637687500 b^{2} c^{3} e-179192721899520 b^{5} c d$

$+339070429687500 c^{3} d f-893019284640000 b^{3} c^{2} f+3183898460400000 b^{2} c f^{2}+34998578496000 b^{4} c^{3}+2965157344800000 b^{2} d e f$

$-3076046938125000 c d^{4}+2049090513750000 b c^{2} e f+11757334963500000 b c d^{2} f-249989846400000 b^{2} c^{2} d^{2}$

$\left.+6684585148828125 c^{2} d^{2} e+253301757187500 b c^{4} d-3137933570625000 b c d e^{2}\right)$

$b_{3}=2^{2} \cdot 3^{3} \cdot 5^{2} \cdot 7^{5}\left(-119352791250000 f^{3} d+923835937500 c^{5} e+2036562500000 c^{2} e^{3}-14145516000000 b^{2} e f^{2}+1453824288000 b^{4} c^{2} e\right.$

$+1935723847680 d b^{5} e+2263282560000 b^{3} c^{3} d+2666558361600 b^{4} c d^{2}+42160986000000 b c^{2} d^{3}+215648793281250 c d^{3} f$

$-6184019520000 d b^{3} e^{2}-11786069531250 c d^{2} e^{2}+3942540000000 d b e^{3}-474054819840 b^{5} c f+7343991562500 b c^{4} f-77308057968750 d^{4} e$

$+2498818359375 c^{4} d^{2}-13526936718750 c^{3} f^{2}+11627929687500 e^{2} f^{2}-19656915468750 b c^{3} d e+11107444950000 b^{2} c d^{2} e$

$+12296954250000 b c d f^{2}-32049232031250 c^{2} d e f-55456852500000 b d^{2} e f+12579405300000 b^{2} c^{2} d f+2222131968000 f^{2} b^{4}$

$\left.-4140860062500 b^{2} c^{2} e^{2}-63207309312 b^{6} c^{2}-807132093750 b^{2} c^{5}-955040625000 b c e^{2} f+4663733760000 b^{3} c e f+13332791808000 f d^{2} b^{3}\right)$

$b_{2}=2 \cdot 3 \cdot 7^{3}\left(13999431398400000 b^{4} d^{2} e-501993771152343750 d^{3} e f+147650253030000000 b^{2} d^{3} f+13999431398400000 b^{5} c^{2} d\right.$

$-70508565618750000 b^{2} d^{2} e^{2}-39373400808000000 b^{3} d f^{2}+640843112109375000 f^{4}-113927664375000000 b d e f^{2}+199373412656250000 b c f^{3}$

$-219625488281250 c^{7}-1121475446191406250 c d^{2} f^{2}-113792664111328125 e c^{2} f^{2}+3874904345088000 b^{6} c e+11248855857421875 b^{2} c^{4} e$

$-4130738964843750 b c^{5} d+12125158565625000 b^{2} c^{3} d^{2}+78746801616000000 b^{3} c d^{3}-13979627676000000 b^{4} c e^{2}+16174336500000000 b^{2} c e^{3}$

$-95569451679744 b^{8} c-4556693232000000 b^{4} c^{4}+357041162460937500 c^{2} d^{4}+5361029296875000 c^{4} e^{2}-204478306347656250 c^{3} d^{2} e$

$-7851484375000000 c e^{4}-216968907487500000 b^{2} c d e f-138232232775000000 b^{3} c^{2} d e-85364120214843750 b c^{3} e f+35873542958400000 b^{4} c d f$

$+54576776362500000 b^{3} c^{3} f+81255806542968750 c^{4} d f-108366909328125000 b^{2} c^{2} f^{2}-93278275207031250 b c d^{3} e$

$\left.-372604495183593750 b c^{2} d^{2} f+146072378320312500 b c^{2} d e^{2}+40730312109375000 d^{2} e^{3}+377268178710937500 c d e^{2} f\right)$ 
$b_{1}=5 \cdot 7^{2}\left(5359375000000000 e^{5}+3951865858007812500 b c d^{2}\right.$ ef $+979586879717376 b^{8} e+275613805656000000 b^{3} f^{3}-9768884765625000 c^{6} e$

$+77017426171875000 c^{5} d^{2}-5554836112896000 b^{6} e^{2}+2321709802734375 b^{2} c^{6}+10970061984000000 b^{4} e^{3}+4103367187500000 c^{3} e^{3}$

$+55159493115234375 c^{4} f^{2}+167336953434000000 b^{3} d^{3} e+29607242411250000 b^{3} c^{4} d-104620750718400000 b^{4} c^{2} d^{2}-1021687018734375000 b c^{3} d^{3}$

$+231488824218750 b^{2} c^{3} e^{2}-229060912500000000 c^{2} d^{2} e^{2}+48223041370560000 b^{5} c^{2} f+11649491191406250 b c^{5} f+12095508728217600 b^{6} d f$

$+6450937988382720 b^{7} c d-6756317381953125000 c^{2} d^{3} f+22785532875000000 b d^{3} e^{2}-152243816457600000 b^{4} c f^{2}-3593187392256000 b^{6} c^{3}$

$-885901518180000000 b^{2} d^{2} f^{2}+5415124297324218750 d^{2} e f^{2}-157134027832031250 c e^{2} f^{2}+797493650625000000 b e f^{3}+20649479586750000 b^{4} c^{3} e$

$+338896825627500000 b^{3} c d e^{2}-179760351093750000 b c d e^{3}-138419377951680000 b^{5} c d e-383861214843750000 d e^{3} f-865138201347656250 c d f^{3}$

$+91935556305468750 b^{2} c^{2} d^{2} e-3249990068554687500 b c^{2} d f^{2}+325453361231250000 b^{2} c e f^{2}+155009007070312500 b c^{4} d e+594702408037500000 b^{2} d e^{2} f$

$-10094490000000000 b^{2} e^{4}-476970373441406250 b^{2} c^{3} d f-1269792176058000000 b^{3} c d^{2} f+632366351367187500 c^{3} d e f$

$\left.+3076046938125000000 c d^{4} e+12118628320312500 b c^{2} e^{2} f-221301775485000000 b^{3} c^{2} e f-160118496619200000 b^{4} d e f\right)$

$b_{0}=3^{2} \cdot 5^{2}\left(27459204328828125 b^{3} c^{3} d e+44149248498600000 b^{4} c d^{2} e+106744394531250 b c^{4} e f+7188851250000000 b c^{3} d e^{2}\right.$

$+440216495145000000 b^{3} d^{2} e f-1196240475937500000 c d^{3} e f-59716324558800000 b^{4} c^{2} d f-516775885605000000 b^{2} c d^{3} f-375961292437500000 b c^{2} d^{3} e$

$-256970176312500000 b d^{2} e^{2} f-379845703125000 b c e^{3} f-183742537104000000 b^{3} c d f^{2}+961264668164062500 b c^{3} d^{2} f-131734136362500000 b^{2} c d^{2} e^{2}$

$+7581112481250000 b^{3} c e^{2} f-18510359293440000 b^{5} c e f+344517257070000000 b^{2} d f^{3}-52917850685952000 b^{5} d^{2} f-5121925300961280 b^{7} d e$

$+27800635693359375 c^{3} e f^{2}+2210908736777343750 c^{2} d^{2} f^{2}-62765703984375000 b^{2} e^{2} f^{2}+19967480859375000 c^{4} d^{2} e+344517257070000000 b^{2} d^{4} e$

$+66351471732000000 b^{4} e f^{2}-59902442578125000 c^{5} d f-11549922890625000 b c^{6} d-14111426849587200 b^{6} c d^{2}-162415278333000000 b^{3} c^{2} d^{3}$

$-1897468822265625 b^{2} c^{5} e+24544373474880000 b^{5} d e^{2}-960461782875000 b^{4} c^{2} e^{2}+1529351464032000 b^{6} c^{2} e+21879112500000000 c d^{2} e^{3}$

$-31295882520000000 b^{3} d e^{3}+1856179335937500 b^{2} c^{2} e^{3}+4456856250000000 b d e^{4}+1254349053296640 b^{7} c f+36053358788671875 b^{2} c^{3} f^{2}$

$-40051757812500 c^{5} e^{2}-334960937500000 c^{2} e^{4}-186912574365234375 c f^{4}+6328909611360000 b^{5} c^{3} d+287652282750000 b^{4} c^{5}+14244213867187500 e^{3} f^{2}$

$-809954488916015625 d e f^{3}-24614001562500000 c^{2} d e^{2} f-4703808949862400 b^{6} f^{2}+379628173828125 c^{8}-284937835587890625 b c d e f^{2}$

$\left.-4141406228203125 b^{3} c^{4} f+92505194627343750 b^{2} c^{4} d^{2}+65342262505078125 b^{2} c^{2} d e f-33110227458984375 b c^{2} f^{3}-83623270219776 b^{8} c^{2}\right)$

$A_{10}=2^{14} \cdot 3^{2} \cdot 5^{2} \cdot 7^{14}\left(682668000 b c e f+41803776 b^{4} c^{2}-1131034375 c^{2} e^{2}+940584960 f^{2} b^{2}-2483460000 f^{2} e-200559375 c^{5}-352836000 b c^{3} d\right.$

$\left.+680486400 b^{2} c^{2} e+836075520 b^{3} d e+7144200000 c d^{2} e-1152900000 b d e^{2}-250822656 b^{3} c f-5292540000 c^{2} d f\right)$

$A_{9}=2^{14} \cdot 3^{2} \cdot 5^{3} \cdot 7^{12} \cdot\left(-38623331250 c d e f-4938071040 b^{2} c d f-14979006000 b c^{2} d e-1332223200 b^{2} c^{4}+6573301875 c^{3} d 2+851175000 c^{4} e\right.$

$-11348859375 d^{2} e^{2}+2492875000 c e^{3}+6697687500 f^{2} c^{2}-658409472 b^{3} c^{2} d+1463132160 b^{4} c e+4938071040 b^{2} d^{2} e-4035150000 b^{2} c e^{2}$

$\left.+3597615000 b c^{3} f-9258883200 f^{2} d b\right)$

$A_{8}=2^{10} \cdot 5 \cdot 7^{10}\left(2624893387200000 b c d^{2} f-846721653750000 b c^{2}\right.$ ef $-1283281211520000 b^{2} d e f+1429956281250000 b c d e^{2}+311098475520000 b^{3} c^{2} f$

$-1036994918400000 b^{3} c d e-49035045427200 b^{4} c^{3}+14158437285888 b^{6} e-58571009280000 b^{4} e^{2}+184454313750000 b c^{4} d+174992892480000 b^{2} c^{2} d^{2}$

$+72369158400000 b^{2} e^{3}-1640558367000000 d^{2} f^{2}-145827410400000 b f^{3}+19812568359375 c^{6}-20460988800000 b^{2} c^{3} e-3106789323750000 c^{2} d^{2} e$

$+229218018750000 c^{3} e^{2}-29292200000000 e^{4}-2916548208000000 b d^{3} e+1515418773750000 c^{3} d f+1476840093750000 d e^{2} f-612475123680000 b^{2} c f^{2}$

$\left.+2253236028750000 \mathrm{cef} f^{2}\right)$

$A_{7}=2^{9} \cdot 3^{2} \cdot 5^{2} \cdot 7^{10}\left(78121827000000 f^{3} d-4266493378560 f^{2} b^{4}-4431306250000 c^{2} e^{3}-26040609000000 d^{4} e+1137731567616 b^{5} c f\right.$

$-1798832812500 c^{5} e-17034451875000 f^{2} e^{2}-29206701562500 f^{2} c^{3}+34342392656250 c d^{2} e^{2}-6461532000000 b d e^{3}+3200928192000 b^{3} c^{3} d$

$-3792438558720 d b^{5} e+10459108800000 d b^{3} e^{2}+6477790725000 b^{2} c^{2} e^{2}+10725230250000 b c^{3} d e-18517766400000 b^{2} c d^{2} e-126414618624 b^{6} c^{2}$

$-1714999910400 b^{4} c^{2} e+793334250000 b c^{4} f+22529949120000 b^{2} e f^{2}-6515380546875 c^{4} d^{2}-20832487200000 b c^{2} d^{3}+884987775000 b^{2} c^{5}$

$\left.-27776649600000 b c d f^{2}+3819170250000 b c e^{2} f-6193164096000 b^{c} e f+61900984687500 c^{2} d e f+114578679600000 b d^{2} e f+20237273280000 b^{2} c^{2} d f\right)$

$A_{6}=2^{9} \cdot 3 \cdot 5^{3} \cdot 7^{8} \cdot\left(853442578125 c^{7}-546852789000000 f^{4}+251989765171200 f^{2} d b^{3}-1806131250000 c^{4} e^{2}+18307625000000 c e^{4}\right.$

$-476054883281250 f^{2} e c^{2}+259612414031250 b c^{3} j 6 f-1781177655600000 b c^{2} d^{2} f+2187411156000000 b c d^{3} e-433879545093750 b c^{2} d e^{2}$

$+1015930959120000 b^{2} c d e f+245638171296000 b^{3} c^{2} d e+10906964409600 b^{4} c^{4}-461407040718750 c^{2} d^{4}-147215171250000 d^{2} e^{3}$

$+298984770000000 c^{3} d^{2} e-285925886820000 b^{2} c^{2} f^{2}-1589290918031250 c d^{2} f^{2}+856736036100000 b c f^{3}+8959636094976 b^{5} c^{2} d-45230724000000 b^{2} c e^{3}$

$+36606880800000 b^{4} c e^{2}-557331321093750 c d e^{2} f-37629282796875 b c^{5} d-109370557800000 b^{2} c^{3} d^{2}-8849023303680 b^{6} c e-34426624050000 b^{2} c^{4} e$

$\left.+44798180474880 b^{4} d^{2} e-49303553040000 b^{3} c^{3} f+62292081796875 c^{4} d f+3349473332625000 d^{3} e f-1130162430600000 b d e f^{2}\right)$

$A_{5}=2^{5} \cdot 5 \cdot 7^{7}\left(1417442429088000000 f^{2} d^{2} b^{2}-12485200000000000 e^{5}+22840481250000000 c^{6} e+83710757812500000 c^{3} e^{3}-31263476428800000 b^{4} e^{3}\right.$

$+125994882585600000 f^{3} b^{3}+843381182109375000 f^{2} e^{2} c+470581317773437500 f^{2} c^{4}-7362005671912500000 b c d^{2} e f+3166863562012500000 b c^{2} d f^{2}$

$-1946795928840000000 b^{2} c e f^{2}-451139485781250000 b c^{2} e^{2} f-1275989841000000000 b^{2} d e^{2} f+731567508840000000 b^{3} c^{2} e f+554377483376640000 b^{4} d e f$

$-865759830468750000 c^{3} d e f-2267907886540800000 b^{3} c d^{2} f+331236546480000000 b^{2} c^{3} d f-1235482227000000000 b^{3} c d e^{2}+763268467500000000 b c d e^{3}$

$+16868450672640000 b^{6} e^{2}+83335976718750000 b c^{4} d e-49997969280000000 b^{2} c^{2} d^{2} e+447981804748800000 b^{5} c d e-159368527080000000 b^{3} c^{4} d$

$-75596929551360000 b^{4} c^{2} d^{2}+2627822955712500000 b c^{3} d^{3}-45171004838400000 b^{4} c^{3} e+2519897651712000000 b^{3} d^{3} e+1538023469062500000 c d^{4} e$

$-3058222453751808 b^{8} e-134394541424640000 b^{5} c^{2} f-201084911015625000 b c^{5} f+6196037403937500000 c^{2} d^{3} f+264589253429760000 b^{4} c f^{2}$

$+8688968085937500 b^{2} c^{6}+894543142500000000 d e^{3} f-22660212444187500000 d^{2} e f^{2}+8920536120562500000 c d f^{3}+364568526000000000 b e f^{3}$

$-49090588903125000 b^{2} c^{3} e^{2}-932669728593750000 c^{2} d^{2} e^{2}-3474793763437500000 b d^{3} e^{2}+14122093083033600 b^{6} c^{3}-262331525654296875 c^{5} d^{2}$

$\left.+25308460800000000 b^{2} e^{4}\right)$ 
$A_{4}=2^{4} \cdot 3^{4} \cdot 5^{2} \cdot 7^{5} \cdot\left(6689861617582080 f^{2} b^{6}-231356250000000 c^{8}-428732586576000000 f^{3} d b^{2}-3309682031250000 c^{5} e^{2}\right.$

$-3433215625000000 c^{2} e^{4}-358872142781250000 f^{4} c+303638323275000000 b c d e f^{2}-451862434170000000 b^{2} c^{2} d e f+148663591501824 b^{8} c^{2}$ $+71562930468750000 c d^{2} e^{3}+116421883200000 b^{4} c^{5}+244588454455078125 c^{3} d^{4}-422283964306640625 d^{4} e^{2}+130661359718400000 b^{3} c d f^{2}$ $+81695043281250000 f^{2} e c^{3}-17965376856000000 b^{3} c e^{2} f-4063263750000000 c^{2} d e^{2} f+620272839375000000 b d^{2} e^{2} f+1415389500000000 b c e^{3} f$ $-12302724000000000 b d e^{4}-43197572742187500 c d^{3}$ ef $+367485074208000000 b^{2} c d^{3} f+14566321953792000 b^{5} c e f-38516912437500000 b c^{4} e f$ $-538978108838400000 b^{3} d^{2} e f+402924173006250000 b c^{3} d^{2} f+17732613104640000 b^{4} c^{2} d f-818506975387500000 b c^{2} d^{3} e$ $-1783963098021888 b^{7} c f+73723926937500000 b c^{3} d e^{2}+138744204007500000 b^{2} c d^{2} e^{2}+2058386904000000 b^{3} c^{3} d e-65330679859200000 b^{4} c d^{2} e$ $-23904956250000000 f^{2} e^{3}+538315584307200 b^{6} c^{2} e+5861394393750000 b^{2} c^{5} e+5946543660072960 b^{7} d e+10913044570312500 c^{4} d^{2} e$ $+30395046528000000 b^{3} d e^{3}-183742537104000000 b^{2} d^{4} e-6338258035200000 b^{4} c^{2} e^{2}-24599823897600000 b^{5} d e^{2}+9851285850000000 b^{2} c^{2} e^{3}$ $-19484805312000000 b^{3} c^{4} f+6963501972656250 c^{5} d f+204183691818750000 b^{2} c^{3} f^{2}-1342922343820312500 c^{2} d^{2} f^{2}$

$-52990440330240000 b^{4} e f^{2}+80130061620000000 b^{2} e^{2} f^{2}-444824236237500000 b c^{2} f^{3}+1913984761500000000 d e f^{3}$

$\left.-7528583107584000 b^{5} c^{3} d+6870810761718750 b c^{6} d-10365609082500000 b^{2} c^{4} d^{2}+97996019788800000 b^{3} c^{2} d^{3}\right)$

$A_{3}=2^{3} \cdot 3 \cdot 5^{3} \cdot 7^{5} \cdot\left(-231356250000000 c^{8}-428732586576000000 f^{3} d b^{2}+6689861617582080 f^{2} b^{6}-3309682031250000 c^{5} e^{2}+97996019788800000 b^{3} c^{2} d^{3}\right.$

$-3433215625000000 c^{2} e^{4}-358872142781250000 f^{4} c+303638323275000000 b c d e f^{2}-451862434170000000 b^{2} c^{2} d e f+148663591501824 b^{8} c^{2}$ $+81695043281250000 f^{2} e c^{3}+116421883200000 b^{4} c^{5}+244588454455078125 c^{3} d^{4}-422283964306640625 d^{4} e^{2}+130661359718400000 b^{3} c d f^{2}$ $+30395046528000000 b^{3} d e^{3}-17965376856000000 b^{3} c e^{2} f-4063263750000000 c^{2} d e^{2} f+620272839375000000 b d^{2} e^{2} f+1415389500000000 b c e^{3} f$ $+71562930468750000 c d^{2} e^{3}-43197572742187500 c d^{3}$ ef $+367485074208000000 b^{2} c d^{3} f+14566321953792000 b^{5} c e f-38516912437500000 b c^{4} e f$ $-538978108838400000 b^{3} d^{2} e f+402924173006250000 b c^{3} d^{2} f+17732613104640000 b^{4} c^{2} d f-818506975387500000 b c^{2} d^{3} e-7528583107584000 b^{5} c^{3} d$ $-12302724000000000 b d e^{4}+73723926937500000 b c^{3} d e^{2}+138744204007500000 b^{2} c d^{2} e^{2}+2058386904000000 b^{3} c^{3} d e-65330679859200000 b^{4} c d^{2} e$ $-23904956250000000 f^{2} e^{3}+538315584307200 b^{6} c^{2} e+5861394393750000 b^{2} c^{5} e+5946543660072960 b^{7} d e+10913044570312500 c^{4} d^{2} e$ $-183742537104000000 b^{2} d^{4} e-6338258035200000 b^{4} c^{2} e^{2}-24599823897600000 b^{5} d e^{2}+9851285850000000 b^{2} c^{2} e^{3}+6870810761718750 b c^{6} d$

$-1783963098021888 b^{7} c f-19484805312000000 b^{3} c^{4} f+6963501972656250 c^{5} d f+204183691818750000 b^{2} c^{3} f^{2}-1342922343820312500 c^{2} d^{2} f^{2}$ $\left.-52990440330240000 b^{4} e f^{2}+80130061620000000 b^{2} e^{2} f^{2}-444824236237500000 b c^{2} f^{3}+1913984761500000000 d e f^{3}-10365609082500000 b^{2} c^{4} d^{2}\right)$

$A_{2}=2^{3} \cdot 7^{3}\left(64873168945312500 c^{9}-18757812500000000000 e^{6}-1339483095488160000000 f^{2} d^{2} b^{4}+26161779208753125000000 f^{4} d b\right.$

$-119065164043392000000 f^{3} b^{5}+49053336016412109375000 f^{3} d^{3}+11537813232421875000 f^{2} e^{3} c-560843682604980468750 f^{2} e c^{4}$

$+29542831787109375000 e^{2} c^{6}-10292009765625000000 e^{4} c^{3}+1910513527976074218750 f^{4} c^{2}+13914190719914625000000 b^{3} c d^{2} e f$ $+6991331335149902343750 b^{2} c^{3} d e f+10034001027852539062500 b c^{2} d e f^{2}-22244465475206542968750 b c d^{2} e^{2} f-6672688981733376000 b^{8} c^{3}$

$-1593990434186718750000 b^{2} c e^{2} f^{2}-32598715496484375000 b^{4} c^{6}+1251360612663574218750 c^{4} d^{4}+1156008087518183424 b^{10} e+98106672032824218750000 d^{6} e$ $+42827801519514375000000 b^{2} d^{2} e f^{2}-7970342942822400000 b^{8} e^{2}+19695990150144000000 b^{6} e^{3}-23916495456000000000 b^{4} e^{4}+23597028000000000000 b^{2} e^{5}$

$+16569799717478027343750 c d e f^{3}-67175712597656250000 b c^{2} e^{3} f-1690686539325000000000 b^{2} d e^{3} f-5985372132203625000000 b^{3} c^{2} d f^{2}$

$-88296004829541796875000 b c d^{3} f^{2}+1839722152753800000000 b^{4} c e f^{2}+71944892824071093750000 b d^{4} e f+852653628126562500000 b^{3} c^{2} e^{2} f$

$+1205810399745000000000 b^{4} d e^{2} f-1042359012597656250000 c^{3} d e^{2} f+46248846347043457031250 c^{2} d^{3} e f-29151696832610625000000 b^{2} c^{2} d^{3} f$

$+62484128808192000000 b^{6} c^{3} e-691331295853800000000 b^{5} c^{2} e f-91760935034179687500 b c^{5}$ ef $-349257814527283200000 b^{6}{ }^{2} e f-1863346193238600000000 b^{4} c^{3} d f$

$+2143172952781056000000 b^{5} c d^{2} f-1497513029475585937500 b c^{4} d^{2} f+12301784590829589843750 b c^{3} d^{3} e+11627457426112500000000 b^{2} c d^{4} e$

$+1167530704515000000000 b^{5} c d e^{2}-361957683691406250000 b c^{4} d e^{2}-5363296110632373046875 b^{2} c^{2} d^{2} e^{2}-1442577403575000000000 b^{3} c d e^{3}$

$+583898814843750000000 b c d e^{4}-780546910549218750000 b^{3} c^{4} d e+2631127508994600000000 b^{4} c^{2} d^{2} e-282228536991744000000 b^{7} c d e$

$+443216854028320312500 c^{2} d^{2} e^{3}-4057248947554687500000 b d^{3} e^{3}+84668561097523200000 b^{7} c^{2} f+566963056184765625000 b^{3} c^{5} f$

$+143385921661376953125 c^{6} d f-147160008049236328125000 c d^{5} f-166691229660748800000 b^{6} c f^{2}+1784515113281250000000 d e^{4} f$

$-2439437968006347656250 b^{2} c^{4} f^{2}-5677469446343994140625 c^{3} d^{2} f^{2}-49947712684749755859375 d^{2} e^{2} f^{2}+3532647655502929687500 b c^{3} f^{3}$

$-996867063281250000000 b e^{2} f^{3}-689034514140000000000 b^{3} e f^{3}+150603258090600000000 b^{5} c^{4} d-40302456207275390625 b c^{7} d$

$+1258810413841845703125 b^{2} c^{5} d^{2}-4966585386296625000000 b^{3} c^{3} d^{3}-9810667203282421875000 b c^{2} d^{5}+47626065617356800000 b^{6} c^{2} d^{2}$

$-13952948911335000000000 b^{2} c d f^{3}+1746549092285156250 b^{2} c^{6} e-685505045269775390625 c^{5} d^{2} e-2381303280867840000000 b^{5} d^{3} e$

$\left.-94370714922093750000 b^{4} c^{3} e^{2}+6567360212896875000000 b^{3} d^{3} e^{2}-15476361157441406250000 c d^{4} e^{2}+15708519052734375000 b^{2} c^{3} e^{3}\right)$

$A_{1}=2 \cdot 3^{2} \cdot 5 \cdot 7^{2}\left(44253777130664062500 b^{2} c^{2} d e^{2} f-1475682251116500000000 b^{3} c d e f^{2}-478911551651367187500 b c d e^{2} f^{2}\right.$

$-10916890583405625000000 b^{2} c d^{3} e f+1488478606379100000000 b^{4} c^{2} d e f+1190651640433920000000 f^{3} d b^{4}-552727184194335937500 c^{3} d f^{3}$

$-21801482673960937500000 f^{4} d^{2}-8720593069584375000000 f^{5} b-43602965347921875000000 d^{5} e f+2054184145279875000000 b^{3} c^{2} f^{3}$

$+65404448021882812500000 c d^{4} f^{2}+85844513334988800000 b^{6} e f^{2}-194716049736600000000 b^{4} e^{2} f^{2}+3457882625756835937500 d e^{2} f^{3}$

$-2884862076345703125000 b c^{3} d^{2} e f-8128181865362227200 f^{2} b^{8}-658479114554625000000 b^{4} c^{3} f^{2}-193790957101875000000 b^{2} c f^{4}$

$-4940454093750000000 b^{2} c^{2} e^{4}-56323365820312500000 c^{4} d^{2} e^{2}+2775771747000000000 b^{4} c^{2} e^{3}-73859963063040000000 b^{5} d e^{3}$

$+116178087375000000000 b^{2} e^{3} f^{2}-29496285000000000000 b d e^{5}-62902448437500000000 c d^{2} e^{4}+59791238640000000000 b^{3} d e^{4}$ 
$+2167515164096593920 b^{9} c f+85627772138160000000 b^{5} c^{4} f-2370465593261718750 b c^{7} f-1709677874091796875000 c^{4} d^{3} f$

$-262287930884765625000 b c^{5} d^{3}+1959173759434752000 b^{8} c^{2} e+1305828038812500000 b^{4} c^{5} e-7225050546988646400 b^{9} d e$

$-65891601562500000 e^{3} c^{5}-4145981297976000000 b^{6} c^{2} e^{2}+769011734531250000000 c^{3} d^{4} e+3973403109375000000 b^{2} c^{5} e^{2}$

$+1190651640433920000000 b^{4} d^{4} e+12196304634286080000 b^{7} c^{3} d-19151182255078125000 b^{3} c^{6} d+124852350865725000000 b^{4} c^{4} d^{2}$

$-6878792970000000000 b^{3} c e^{3} f+1453432178264062500000 b^{2} c^{3} d^{4}-6394981327382812500 b^{3} c^{3} d e^{2}-1066855106060100000000 b^{4} c d^{2} e^{2}$

$+797493650625000000000 d^{4} e^{3}+39851714714112000000 b^{7} d e^{2}-238130328086784000000 b^{5} c^{2} d^{3}+1102250152828125000000 b c^{2} d^{3} e^{2}$

$-1785977460650880000000 b^{4} c d^{3} f-18582594433593750000 b c^{3} d e^{3}+553815035156250000000 b^{2} c d^{2} e^{3}-132600864276720000000 b^{5} c^{3} d e$

$+26161779208753125000000 b c^{2} d^{4} f+282228536991744000000 b^{6} c d^{2} e-497533299649804687500 b^{2} c^{4} d^{2} e-21180712531289062500 b^{3} c^{4} e f$

$-23597441565143040000 b^{7} c e f+1309716804477312000000 b^{5} d^{2} e f-70996261920117187500 b^{2} c^{5} d f-20301909791625000000 b^{3} c^{3} d^{2} f$

$+47673706069335937500 b c^{6} d e+1824711043695750000000 b^{3} c^{2} d^{3} e-14534321782640625000000 b c d^{5} e+43001246777343750000 c^{2} d e^{3} f$

$+182886113012695312500 c^{5} d e f+12458939062500000000 b c e^{4} f-317507104115712000000 b^{5} c d f^{2}-526406842089843750000 b c^{4} d f^{2}$

$-134562534601420800000 b^{6} c^{2} d f+43655865760080000000 b^{5} c e^{2} f-8187922968750000000 b c^{4} e^{2} f-3014525999362500000000 b^{3} d^{2} e^{2} f$

$+2113358174156250000000 b d^{2} e^{3} f+16929024323970937500000 b^{2} c^{2} d^{2} f^{2}+2093420832890625000000 c d^{3} e^{2} f+280368861547851562500 f^{4} e c$

$-8440672798215000000000 b^{2} d e f^{3}-5262924058198242187500 c^{2} d^{2} e f^{2}-26161779208753125000000 b d^{3} e f^{2}+23254914852225000000000 b c d^{2} f^{3}$

$-3749222721384000000 b^{6} c^{5}+968815905029296875 b^{2} c^{8}+9808823144531250000 c^{7} d^{2}-65051289843750000000 f^{2} e^{2} c^{3}$

$+1339843750000000000 e^{5} c^{2}-128892537789257812500 b^{2} c^{3} e f^{2}+863536093567382812500 b c^{2} e f^{3}-144501010939772928 b^{10} c^{2}$

-1326295898437500000 e $\left.c^{8}+10830575555419921875 f^{2} c^{6}-56976855468750000000 f^{2} e^{4}\right)$

$A_{0}=3^{4} \cdot 5^{3}\left(+733534659844875000000 b^{2} c d^{2} e f^{2}-48264980313867187500 b c^{3} d e f^{2}-3537852491250000000 b^{2} c d e^{3} f\right.$

$-245037899091037500000 b^{3} c^{2} d^{2} e f+53368632376980480000 b^{6} c d e f+6304788593085937500 b^{2} c^{4} d e f+2260894499521875000000 b c d^{4}$ ef

$+12974633789062500 c^{10}-595795183593750000 b c^{8} d-1076935425804000000 b^{5} c^{5} d+252876769144602624 b^{9} c^{2} d-14469724796940000000 b^{4} c d e^{2} f$

$+367569090000000000 b^{2} d^{2} e^{4}+9119299206445312500 b^{2} c^{6} d^{2}+1675731938388480000 b^{6} c^{3} d^{2}-54415270335431250000 b^{3} c^{4} d^{3}+104182018537968000000 b^{4} c^{2} d^{4}$

$+93480771081600000 b^{6} c^{4} e-129317694433593750 b^{2} c^{7} e-1041820185379680000000 b^{3} d^{5} e+1017211289062500000 c^{6} d^{2} e+51837087290625000000 b c^{2} d^{2} e^{2} f$

$+7586303074338078720 b^{8} d^{2} e-104702981241796875 b^{4} c^{4} e^{2}-34870250374848000000 b^{6} d^{2} e^{2}+39697461720000000000 b^{4} d^{2} e^{3}$

$+125019882421875000 b^{2} c^{4} e^{3}-1369573279593750000 b^{3} c^{6} f+2704690146170880000 b^{7} c^{3} f-124054567875000000000 d^{3} e^{3} f-27840669221998080000 b^{6} c^{2} f^{2}$

$+2187911250000000000 c^{3} d^{2} e^{3}+14224318264383897600 b^{7} d f^{2}+6953147766386718750 b^{2} c^{5} f^{2}+222692981458007812500 c^{4} d^{2} f^{2}+2421516357421875000 c^{2} e^{3} f^{2}$

$+3391341749282812500000 d^{4} e f^{2}+1928463068847656250 c^{5} e f^{2}-5767588008984375000 b c^{4} f^{3}+129648734180582400000 b^{5} c f^{3}$

$-5087012623924218750000 c d^{3} f^{3}-520910092689840000000 b^{3} d^{2} f^{3}+167473666631250000000 b^{2} e f^{4}+100530991698046875 b^{4} c^{7}$

$-99825278824857600 b^{8} c^{4}+3488378906250000 c^{7} e^{2}-16748046875000000 c^{4} e^{4}+1134472500000000000 d^{2} e^{5}-231515596751040000000 b^{4} f^{4}$

$-25233197539306640625 c^{3} f^{4}+1695670874641406250000 d f^{5}-30286759735107421875 e^{2} f^{4}-4426806867259392000 b^{7} c^{2} d e+4711911063398437500 b^{3} c^{5} d e$

$-33992369364240000000 b^{4} c^{3} d^{2} e-38735405887500000000 b c^{4} d^{3} e+61737492466944000000 b^{5} c d^{3} e+411267475627312500000 b^{2} c^{2} d^{4} e$

$+154325882812500000 b c^{5} d e^{2}+2198388719394000000 b^{5} c^{2} d e^{2}-10588943472187500000 b^{2} c^{3} d^{2} e^{2}+164347491520800000000 b^{3} c d^{3} e^{2}$

$-4782117619125000000 b^{3} c^{2} d e^{3}-41351522625000000000 b c d^{3} e^{3}+417323812500000000 b c^{2} d e^{4}-3793151537169039360 b^{8} c d f$

$+5780233979730000000 b^{4} c^{4} d f+98903453635546875000 b c^{5} d^{2} f-23813032808678400000 b^{5} c^{2} d^{2} f-842990663393156250000 b^{2} c^{3} d^{3} f$

$+1562730278069520000000 b^{3} c d^{4} f+42383803710937500 b c^{6} e f-844705274742000000 b^{5} c^{3} e f-119624047593750000000 c^{3} d^{3} e f$

$-578788991877600000000 b^{4} d^{3} e f+1553266568601562500 b^{3} c^{3} e^{2} f-11395371093750000 b c^{3} e^{3} f-53591573322000000000 b^{2} d^{3} e^{2} f$

$-3486983554687500000 c^{4} d e^{2} f-2127135937500000000 c d e^{4} f+8254059283968750000 b^{3} c^{3} d f^{2}-3730475924211093750000 b c^{2} d^{3} f^{2}$

$+659819450740464000000 b^{4} c d^{2} f^{2}+3508972062750000000 b^{4} c^{2} e f^{2}-191386226647526400000 b^{5} d e f^{2}+154968966189450000000 b^{3} d e^{2} f^{2}$

$-8247228898535156250 b^{2} c^{2} e^{2} f^{2}+209342083289062500000 c d^{2} e^{2} f^{2}-12060860765625000000 b d e^{3} f^{2}-425263489195781250000 b^{2} c^{2} d f^{3}$

$-4845881557617187500 b c e^{2} f^{3}+678268349856562500000 b d^{2} e f^{3}-133330969713867187500 c^{2} d e f^{3}-33494733326250000000 b^{3} c e f^{3}$

$\left.-3051633867187500000 c^{7} d f+791313074832656250000 b c d f^{4}\right)$

Department of Mathematics \& Statistics, Oakland University, Rochester, Mi, 48309.

E-mail address: shaska@oakland.edu 